\title{
Summability Methods for Elastic Local Fields in Periodic Heterogeneous Materials
}

\author{
Andrea Caporale $(10$ \\ Department of Civil and Mechanical Engineering, University of Cassino and Southern Lazio, \\ Via G. Di Biasio 43, 03043 Cassino, FR, Italy \\ Correspondence should be addressed to Andrea Caporale; a.caporale@unicas.it
}

Received 10 July 2018; Accepted 3 October 2018; Published 25 October 2018

Academic Editor: Fabrizio Greco

Copyright (C) 2018 Andrea Caporale. This is an open access article distributed under the Creative Commons Attribution License, which permits unrestricted use, distribution, and reproduction in any medium, provided the original work is properly cited.

In the past, Eshelby's method for a single inclusion in an infinite medium has been extended to periodic heterogeneous materials for the evaluation of global properties. In this work, the extended Eshelby's method is used for the evaluation of local fields such as strain and stress in periodic heterogeneous materials characterized by unit cells containing multiple fibres or voids with different geometric and mechanical properties. The proposed method provides Fourier coefficients that are used to construct partial sums of three-dimensional trigonometric Fourier series of local fields. These partial sums exhibit unwanted effects such as the Gibbs phenomenon. In order to attenuate these effects, the behaviour of iterated Fejér partial sums and means of the Riesz summability method is investigated. Extensive numerical examples on both a multiphase composite and a material with voids are provided: in the examples, partial sums, iterated Fejér partial sums, and Riesz means for the local stress are compared with FEM solutions. The numerical comparison shows Riesz means perform better than partial sums and iterated Fejér partial sums and are effective in approximating elastic local fields in periodic heterogeneous solids.

\section{Introduction}

The Fourier series are widely used in engineering $[1,2]$. In the mechanics of solids and structures, Fourier series are frequently adopted for finding numerical and analytic solutions [3-5]. Geometric, mechanical properties, and local fields in heterogeneous materials with periodic microstructure seem to be naturally suited to being represented with periodic functions, but Fourier series and relevant partial sums exhibit large oscillations, known as Gibbs phenomenon, at the jump discontinuity of the field they represent. It is noted that the subdivision of the domain in smaller subdomains, as adopted in the Finite Element Method (FEM), favours the representation of jump discontinuity so that one can find many FEM applications $[6,7]$ in many scientific disciplines. Many authors $[8,9]$ have successfully used partial sums of Fourier series for determining global (averaged) properties of periodic composite materials. Specifically, Eshelby's method introduced for a single inclusion in an infinite medium has been extended to the periodic heterogeneous materials for the evaluation of global properties. Few works $[10,11]$ have tried to use Fourier series for determining local fields such as strain and stress distribution in phases of composite materials and have observed anomalous large oscillations due to the Gibbs phenomenon. Recently, Caporale et al. [12] have applied summability methods in order to reduce the Gibbs phenomenon in unidirectional composites characterized by a simple geometry. In this work, the extended Eshelby's method is used for determining the Fourier coefficients of the local strain in periodic heterogeneous materials with more complex microstructure and summability methods are applied in order to reduce the Gibbs phenomenon. Section 2 deals with Eshelby's method extended to periodic materials for the evaluation of local fields. The means of the summability methods adopted in this work are briefly described in Section 3. Extensive numerical examples on both a multiphase composite and a material with voids are provided in Section 4: in these examples, partial sums, iterated Fejér partial sums, and Riesz means for the local stress are compared with solutions provided by accurate FEM analyses. 


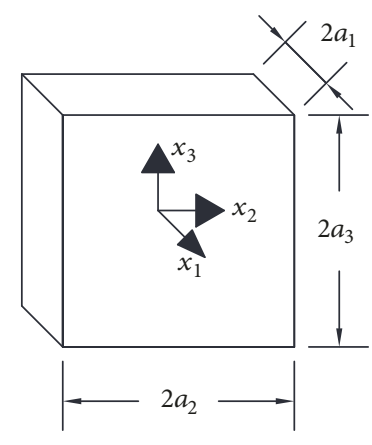

(a)

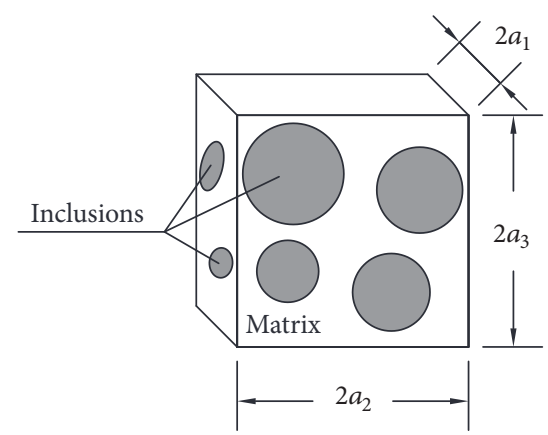

(b)

FIGURE 1: (a) Boundary of the unit cell and adopted coordinate system; (b) unit cell containing several inclusions.

\section{Partial Sums of Fourier Series via Eshelby's Method Extended to Periodic Solids}

The consideration is focused on an infinite periodic heterogeneous material, i.e., an infinitely extended solid that can be obtained by the infinite repetition of a representative unit cell along the $x_{1^{-}}, x_{2^{-}}$, and $x_{3}$-axes of a Cartesian coordinate system. Figure 1(a) shows the boundary of the unit cell and the adopted coordinate system. The centre of the unit cell coincides with origin of the coordinate system. Figure 1(b) shows the unit cell with several inclusions, which may be fibres, particles, voids, defects, and so on. The dimensions of the unit cell along the $x_{1^{-}}, x_{2^{-}}$, and $x_{3}$-axes of the coordinate system are denoted by $2 a_{1}, 2 a_{2}$, and $2 a_{3}$, respectively. In this work, it is assumed that $a_{1}=a_{2}=a_{3}=\pi$, but the proposed formulation can be easily extended to unit cell with arbitrary dimensions. Because of periodicity, entities defining the geometry, the mechanical properties, and the local fields (such as strain and stress) of the considered material are defined by periodic functions. Here, Fourier series representations are adopted for the above-mentioned variables. Next, $\mathbb{Z}$ and $\mathbb{N}$ denote the set of integers and nonnegative integers, respectively, and $\mathrm{i}=\sqrt{-1}$. The geometric and mechanical properties are defined by the following fourth-order tensor:

$$
\mathbf{C}(\mathbf{x})=\sum_{\zeta \in \mathbb{Z}^{3}} \widehat{\mathbf{C}}(\zeta) e^{\mathrm{i} \zeta \cdot \mathbf{x}}
$$

where $\mathbf{x}=\left(x_{1}, x_{2}, x_{3}\right) \in \mathbb{T}^{3}$ with $\mathbb{T}:=[-\pi, \pi], \zeta=\left(\zeta_{1}, \zeta_{2}, \zeta_{3}\right)$ and $\zeta \cdot \mathbf{x}=\zeta_{1} \mathrm{x}_{1}+\zeta_{2} \mathrm{x}_{2}+\zeta_{3} \mathrm{x}_{3}$. Tensor $\mathbf{C}(\mathbf{x})$ also represents the local elasticity of the considered material. The Fourier coefficient $\widehat{\mathbf{C}}(\zeta)$ is

$$
\widehat{\mathbf{C}}(\boldsymbol{\zeta})=\frac{1}{(2 \pi)^{3}} \int_{\mathbb{T}^{3}} \mathbf{C}(\mathbf{x}) e^{-\mathrm{i} \zeta \cdot \mathbf{x}} d \mathbf{x}
$$

Closed-form expressions of the Fourier coefficients (2) for different types of heterogeneous materials can be found in [11]. The first aim of this work is to determine local fields, such as strain and stress, in heterogeneous materials subject to given loads in the framework of linear elasticity. Therefore, the considered strain $\boldsymbol{\varepsilon}$ is the infinitesimal strain tensor, which is equal to the symmetric part of the displacement gradient; and the considered stress $\boldsymbol{\sigma}$ is the Cauchy stress tensor. Both $\boldsymbol{\varepsilon}$ and $\boldsymbol{\sigma}$ are second-order symmetric tensors and are functions of the spacial variable $\mathbf{x}$, which represents a point of the unit cell. Under these assumptions, $\boldsymbol{\varepsilon}$ and $\boldsymbol{\sigma}$ appear as local fields in contrast with global entities (such as the overall properties of the material) which are averages over the volume of the unit cell. The local strain is given by

$$
\boldsymbol{\varepsilon}(\mathbf{x})=\sum_{\zeta \in \mathbb{Z}^{3}} \widehat{\boldsymbol{\varepsilon}}(\boldsymbol{\zeta}) e^{\mathrm{i} \zeta \cdot \mathbf{x}}
$$

where the Fourier coefficient $\widehat{\boldsymbol{\varepsilon}}(\zeta)$ is

$$
\widehat{\boldsymbol{\varepsilon}}(\boldsymbol{\zeta})=\frac{1}{(2 \pi)^{3}} \int_{\mathbb{T}^{3}} \boldsymbol{\varepsilon}(\mathbf{x}) e^{-\mathrm{i} \boldsymbol{\zeta} \cdot \mathbf{x}} d \mathbf{x}
$$

The average of the strain (3) over the unit cell is

$$
\boldsymbol{\varepsilon}_{0}=\widehat{\boldsymbol{\varepsilon}}(\mathbf{0})=\frac{1}{(2 \pi)^{3}} \int_{\mathbb{T}^{3}} \boldsymbol{\varepsilon}(\mathbf{x}) d \mathbf{x}
$$

Therefore, the local strain is represented as the addition of the average strain $\boldsymbol{\varepsilon}_{0}$ and the periodic part $\boldsymbol{\varepsilon}_{p}$ of the local strain:

$$
\boldsymbol{\varepsilon}(\mathbf{x})=\varepsilon_{0}+\varepsilon_{p}(\mathbf{x})
$$

with

$$
\boldsymbol{\varepsilon}_{p}(\mathbf{x})=\sum_{\zeta \in \mathbb{Z}^{3}, \zeta \neq \mathbf{0}} \widehat{\boldsymbol{\varepsilon}}_{p}(\zeta) e^{\mathrm{i} \zeta \cdot \mathbf{x}}
$$

and

$$
\widehat{\boldsymbol{\varepsilon}}_{p}(\boldsymbol{\zeta})=\widehat{\boldsymbol{\varepsilon}}(\boldsymbol{\zeta}) \quad \forall \boldsymbol{\zeta} \neq \mathbf{0}
$$

Next, local strain and stress are evaluated in a periodic heterogeneous material subject to a given average strain $\boldsymbol{\varepsilon}_{0}$. According to Eshelby's method, a homogeneous material equivalent to the considered periodic heterogeneous material is taken into account. The equivalence is made possible by imposing on the homogeneous material an eigenstrain $\boldsymbol{\varepsilon}^{*}(\mathbf{x})$, which is the unknown of the problem. The relation imposing this equivalence is the following consistency condition:

$$
\begin{array}{r}
\mathbf{C}(\mathbf{x}):\left(\boldsymbol{\varepsilon}_{0}+\boldsymbol{\varepsilon}_{p}(\mathbf{x})\right)=\mathbf{C}^{(R)}:\left(\boldsymbol{\varepsilon}_{0}+\boldsymbol{\varepsilon}_{p}(\mathbf{x})-\boldsymbol{\varepsilon}^{*}(\mathbf{x})\right) \\
\forall \mathbf{x} \in \mathbb{T}^{3}
\end{array}
$$

where $\mathbf{C}^{(R)}$ is the constant elasticity of the above-mentioned homogeneous material. The eigenstrain admits the Fourier series representation 


$$
\boldsymbol{\varepsilon}^{*}(\mathbf{x})=\sum_{\zeta \in \mathbb{Z}^{3}} \widehat{\boldsymbol{\varepsilon}}^{*}(\boldsymbol{\zeta}) e^{\mathrm{i} \zeta \cdot \mathbf{x}}
$$

In order to solve (9), coefficients $\widehat{\boldsymbol{\varepsilon}}_{p}(\boldsymbol{\zeta})$ for $\boldsymbol{\zeta} \neq \mathbf{0}$ are expressed in terms of $\widehat{\boldsymbol{\varepsilon}}^{*}(\boldsymbol{\zeta})$ through the following relation obtained by solving the equilibrium equation of the homogeneous material with elasticity $\mathbf{C}^{(R)}$ subject to a periodic eigenstrain $\boldsymbol{\varepsilon}^{*}(\mathbf{x})$ [8]:

$$
\widehat{\boldsymbol{\varepsilon}}_{p}(\zeta)=\widehat{\mathbf{S}}_{p}(\zeta): \widehat{\boldsymbol{\varepsilon}}^{*}(\zeta)
$$

Taking into account (11), the consistency condition (9) becomes

$$
\begin{array}{r}
\sum_{\boldsymbol{\zeta} \in \mathbb{Z}^{3}} \widehat{\mathbf{C}}(\boldsymbol{\zeta}) e^{\mathrm{i} \zeta \cdot \mathbf{x}}:\left(\boldsymbol{\varepsilon}_{0}+\sum_{\boldsymbol{\eta} \in \mathbb{Z}^{3}, \boldsymbol{\eta} \neq \mathbf{0}} \widehat{\boldsymbol{S}}_{p}(\boldsymbol{\eta}): \widehat{\boldsymbol{\varepsilon}}^{*}(\boldsymbol{\eta}) e^{\mathrm{i} \boldsymbol{\eta} \cdot \mathbf{x}}\right) \\
=\mathbf{C}^{(R)}: \\
\left(\boldsymbol{\varepsilon}_{0}+\sum_{\zeta \in \mathbb{Z}^{3}, \boldsymbol{\zeta} \neq \mathbf{0}} \widehat{\mathbf{S}}_{p}(\boldsymbol{\zeta}): \widehat{\boldsymbol{\varepsilon}}^{*}(\boldsymbol{\zeta}) e^{\mathrm{i} \boldsymbol{\zeta} \cdot \mathbf{x}}-\sum_{\boldsymbol{\zeta} \in \mathbb{Z}^{3}} \widehat{\boldsymbol{\varepsilon}}^{*}(\boldsymbol{\zeta}) e^{\mathrm{i} \zeta \cdot \mathbf{x}}\right) \\
\forall \mathbf{x} \in \mathbb{T}^{3}
\end{array}
$$

Relation (12) provides a square system of equations in the unknowns $\widehat{\boldsymbol{\varepsilon}}^{*}(\boldsymbol{\zeta})$ if the infinite Fourier series in (12) are truncated. After solving this system imposing the truncation $\left|\zeta_{i}\right|,\left|\eta_{i}\right| \leq n(i=1,2,3)$ with $n \in \mathbb{N}, n<\infty$, and $\boldsymbol{\eta} \neq \mathbf{0}$, all the coefficients $\widehat{\boldsymbol{\varepsilon}}^{*}(\zeta)$ such that $\left|\zeta_{i}\right| \leq n(i=1,2,3)$ with $n \in \mathbb{N}$ and $n<\infty$ are known [11]. From (8) and (11), the Fourier coefficients $\widehat{\boldsymbol{\varepsilon}}_{p}(\boldsymbol{\zeta})$ and $\widehat{\boldsymbol{\varepsilon}}(\boldsymbol{\zeta})$ are also known for $\zeta \neq \mathbf{0}$ and $\left|\zeta_{i}\right| \leq n(i=1,2,3)$ with $n \in \mathbb{N}$ and $n<\infty$. Finally, it is possible to build the following $n$th rectangular partial sum $(n \in \mathbb{N})$ for the strain (given an integrable function $f(x)$ with $x \in \mathbb{R}$, the $n$th partial sum of the Fourier series of $f(x)$ is denoted by $s_{n} f(x)$ in mathematical literature. This convention is also used in this work, where the $n$th rectangular partial sum of the Fourier series of the strain tensor $\boldsymbol{\varepsilon}(\mathbf{x})$ is denoted by $s_{n} \boldsymbol{\varepsilon}(\mathbf{x})$. Both $\boldsymbol{\varepsilon}(\mathbf{x})$ and $s_{n} \boldsymbol{\varepsilon}(\mathbf{x})$ are second-order tensors and the $i j$-component of $s_{n} \boldsymbol{\varepsilon}(\mathbf{x})$ is denoted by $s_{n} \varepsilon_{i j}(\mathbf{x})$. Similar notations can be found in scientific literature for other operators: for example, in [8], $F \boldsymbol{\varepsilon}(\boldsymbol{\zeta})$ denotes a Fourier coefficient of $\boldsymbol{\varepsilon}(\mathbf{x})$ and its $i j$-component is denoted by $\left.F \varepsilon_{i j}(\zeta)\right)$ :

$$
s_{n} \boldsymbol{\varepsilon}(\mathbf{x})=\sum_{\left|\zeta_{1}\right| \leq n} \sum_{\left|\zeta_{2}\right| \leq n} \sum_{\left|\zeta_{3}\right| \leq n} \widehat{\boldsymbol{\varepsilon}}(\boldsymbol{\zeta}) e^{\mathrm{i} \zeta \cdot \mathbf{x}}
$$

Formula (13) represents the following definition:

$$
s_{n} \varepsilon_{i j}(\mathbf{x})=\sum_{\left|\zeta_{1}\right| \leq n} \sum_{\left|\zeta_{2}\right| \leq n} \sum_{\left|\zeta_{3}\right| \leq n} \widehat{\varepsilon}_{i j}(\zeta) e^{\mathrm{i} \zeta \cdot \mathbf{x}}
$$

where the scalar $s_{n} \varepsilon_{i j}(\mathbf{x})$ is the $n$th rectangular partial sum of the $i j$-component of the local strain tensor $\boldsymbol{\varepsilon}(\mathbf{x})$. Next, the expressions of the local stress $\boldsymbol{\sigma}(\mathbf{x})$ in periodic heterogeneous material subject to a given average strain $\boldsymbol{\varepsilon}_{0}$ are provided. In Eshelby's method, the local stress $\boldsymbol{\sigma}(\mathbf{x})$ can be evaluated on the heterogeneous material or on the equivalent homogeneous material. The following relation provides the local stress on the equivalent homogeneous material:

$$
\boldsymbol{\sigma}(\mathbf{x})=\mathbf{C}^{(R)}:\left(\boldsymbol{\varepsilon}_{0}+\boldsymbol{\varepsilon}_{p}(\mathbf{x})-\boldsymbol{\varepsilon}^{*}(\mathbf{x})\right) \quad \forall \mathbf{x} \in \mathbb{T}^{3}
$$

A first approximation of the local stress $\boldsymbol{\sigma}(\mathbf{x})$ is obtained by replacing the periodic strain and eigenstrain in (15) with the corresponding partial sums. The resulting stress is

$$
s_{n} \boldsymbol{\sigma}(\mathbf{x})=\mathbf{C}^{(R)}:\left(s_{n} \boldsymbol{\varepsilon}(\mathbf{x})-s_{n} \boldsymbol{\varepsilon}^{*}(\mathbf{x})\right) \quad \forall \mathbf{x} \in \mathbb{T}^{3}
$$

where

$$
s_{n} \boldsymbol{\varepsilon}^{*}(\mathbf{x})=\sum_{\left|\zeta_{1}\right| \leq n} \sum_{\left|\zeta_{2}\right| \leq n} \sum_{\left|\zeta_{3}\right| \leq n} \widehat{\boldsymbol{\varepsilon}}^{*}(\boldsymbol{\zeta}) e^{\mathrm{i} \zeta \cdot \mathbf{x}}
$$

Rewriting formula (16) in index notation, one obtains

$$
s_{n} \sigma_{i j}(\mathbf{x})=C_{i j k l}^{(R)}:\left(s_{n} \varepsilon_{k l}(\mathbf{x})-s_{n} \varepsilon_{k l}^{*}(\mathbf{x})\right) \quad \forall \mathbf{x} \in \mathbb{T}^{3}
$$

where the scalar $s_{n} \sigma_{i j}(\mathbf{x})$ is the $n$th rectangular partial sum of the $i j$-component of the local stress tensor $\boldsymbol{\sigma}(\mathbf{x})$. In Section 4, the partial sum stress (16) is plotted for two different types of periodic solids and is compared with the solution provided by the Finite Element Method (FEM). This comparison between partial sum stress and FEM solution shows that the partial sum stress is not adequate to describe accurately the local stress in heterogeneous solids: in fact, the Gibbs phenomenon occurs at the jump discontinuity of the local field and does not vanish by increasing $n$ in the rectangular partial sums. In order to overcome this difficulty, means of summability methods are introduced in Section 3.

\section{Means of the Summability Methods for Local Fields in Periodic Solids}

Summability methods are known to provide means with convergence properties better than those of the original partial sums, such as (13). In the last decade, many authors [13-17] have demonstrated the convergence properties of the means of the partial sums of the multidimensional trigonometric Fourier series. An aim of this work is to show the behaviour of the summability means in an area of the mechanics of solids. Next, Fejér and Riesz means together with the iterated Fejér partial sums [18] for the local strain are described and then used for the evaluation of local stress in periodic heterogeneous materials. In-depth details on convergence properties of the summability means adopted in this work can be found in [17]. The $n$th $\ell_{q}$-Fejér mean $(n \in \mathbb{N})$ of the local strain $\boldsymbol{\varepsilon}(\mathbf{x})$ is defined by

$$
\sigma_{n}^{q} \boldsymbol{\varepsilon}(\mathbf{x})=\sum_{\zeta \in \mathbb{Z}^{3},\|\zeta\|_{q} \leq n}\left(1-\frac{\|\zeta\|_{q}}{n}\right) \widehat{\boldsymbol{\varepsilon}}(\boldsymbol{\zeta}) e^{\mathrm{i} \zeta \cdot \mathbf{x}}
$$

Analogously to Section 2, the left-hand side of (19) is a second-order tensor, whose $i j$-component is $\sigma_{n}^{q} \varepsilon_{i j}(\mathbf{x})$ and represents the $n$th $\ell_{q}$-Fejér mean of $i j$-component of the local strain tensor $\boldsymbol{\varepsilon}(\mathbf{x})$. A generalization of the mean (19) is the following $n$th $\ell_{q}$-Riesz mean $(n \in \mathbb{N})$ of the local strain $\boldsymbol{\varepsilon}(\mathbf{x})$ :

$$
\sigma_{n}^{q, \alpha} \boldsymbol{\varepsilon}(\mathbf{x})=\sum_{\zeta \in \mathbb{Z}^{3},\|\zeta\|_{q} \leq n}\left(1-\left(\frac{\|\zeta\|_{q}}{n}\right)^{\gamma}\right)^{\alpha} \widehat{\boldsymbol{\varepsilon}}(\boldsymbol{\zeta}) e^{\mathrm{i} \zeta \cdot \mathbf{x}}
$$

where (in this work, the conceptual difference between partial sums and summability means is highlighted by the adoption 
TABLE 1: Geometrical and mechanical properties of the fibres of the multiphase composite.

\begin{tabular}{|c|c|c|c|c|c|}
\hline$i$ & $x_{2}^{(i)} /(2 \pi)$ & $x_{3}^{(i)} /(2 \pi)$ & $\rho_{i} /(2 \pi)$ & $E_{i}(\mathrm{GPa})$ & $v_{i}$ \\
\hline 1 & 0.12 & 0.12 & 0.09 & 5.0 & 0.2 \\
\hline 2 & 0.35 & 0.16 & 0.10 & 4.5 & 0.2 \\
\hline 3 & 0.20 & 0.35 & 0.08 & 4.0 & 0.2 \\
\hline 4 & -0.12 & 0.12 & 0.09 & 5.0 & 0.2 \\
\hline 5 & -0.35 & 0.16 & 0.10 & 4.5 & 0.2 \\
\hline 6 & -0.20 & 0.35 & 0.08 & 4.0 & 0.2 \\
\hline 7 & -0.12 & -0.12 & 0.09 & 5.0 & 0.2 \\
\hline 8 & -0.35 & -0.16 & 0.10 & 4.5 & 0.2 \\
\hline 9 & -0.20 & -0.35 & 0.08 & 4.0 & 0.2 \\
\hline 10 & 0.12 & -0.12 & 0.09 & 5.0 & 0.2 \\
\hline 11 & 0.35 & -0.16 & 0.10 & 4.5 & 0.2 \\
\hline 12 & 0.20 & -0.35 & 0.08 & 4.0 & 0.2 \\
\hline
\end{tabular}

of appropriate symbols. For example, $s_{n}$ is used to denote partial sums (see formula (13)) and $\sigma_{n}^{q, \alpha}$ is used for Riesz summability means (see formula (20)), as also done in mathematical literature [17]) $0 \leq \alpha<\infty$ and $1 \leq \gamma<\infty$. Finally, the iterated Fejér partial sum of the Fourier series (3) is

$$
\begin{aligned}
\sigma_{n} \boldsymbol{\varepsilon}(\mathbf{x}) & =\sum_{\left|\zeta_{1}\right| \leq n} \sum_{\left|\zeta_{2}\right| \leq n} \sum_{\left|\zeta_{3}\right| \leq n}\left(1-\frac{\left|\zeta_{1}\right|}{n+1}\right)\left(1-\frac{\left|\zeta_{2}\right|}{n+1}\right) \\
& \left(1-\frac{\left|\zeta_{3}\right|}{n+1}\right) \widehat{\boldsymbol{\varepsilon}}(\zeta) e^{\mathrm{i} \zeta \cdot \mathbf{x}}
\end{aligned}
$$

As done at the end of Section 2, approximations of local stress are obtained by replacing periodic strain and eigenstrain in (15) with the relevant Riesz means or iterated Fejér partial sums. Therefore, the Riesz approximation of the local stress is

$$
\sigma_{n}^{q, \alpha} \boldsymbol{\sigma}(\mathbf{x})=\mathbf{C}^{(R)}:\left(\sigma_{n}^{q, \alpha} \boldsymbol{\varepsilon}(\mathbf{x})-\sigma_{n}^{q, \alpha} \boldsymbol{\varepsilon}^{*}(\mathbf{x})\right) \quad \forall \mathbf{x} \in \mathbb{T}^{3}
$$

and the iterated Fejér partial sum stress is

$$
\sigma_{n} \boldsymbol{\sigma}(\mathbf{x})=\mathbf{C}^{(R)}:\left(\sigma_{n} \boldsymbol{\varepsilon}(\mathbf{x})-\sigma_{n} \boldsymbol{\varepsilon}^{*}(\mathbf{x})\right) \quad \forall \mathbf{x} \in \mathbb{T}^{3}
$$

In Section 4, the approximations (22) and (23) are plotted for two different types of periodic solids and are compared with the solution provided by FEM.

\section{Results and Discussion}

The methods proposed in the previous sections are applied here to calculate the local stress in two different types of heterogeneous materials: the first type is a composite material containing long fibres with various geometrical and mechanical properties; the second type is a material containing circular voids with various sizes. In both applications, the behaviour of the partial sums (of the Fourier series) is first investigated, observing that these are not adequate to represent the local fields especially at the jump discontinuities of the local fields. The improvement provided by the iterated Fejér partial sums is also shown. Finally, the Riesz means are used, showing that they exhibit better convergence properties than those of the original partial sums of Fourier series.
4.1. Multiphase Composite. In the first numerical example, a unidirectional composite with unit cell containing multiple fibres with various sizes and various mechanical properties is considered. The origin of the Cartesian coordinate system is located at the centre of the unit cell. The lengths of the unit cell are given by $2 a_{1}=2 a_{2}=2 a_{3}=2 \pi$; the unit of the lengths is not specified as the next results do not depend on it. The fibres are infinitely long right circular cylinders whose axes are parallel to the $x_{1}$-axis of the coordinate system. The unit cell contains $N_{f}=12$ fibres. The generic fibre and its volume are denoted by $\Omega_{i}$ for $i=1, \ldots, N_{f}$. The intersection of a generic volume $\Omega_{i}$ with plane $x_{1}=0$ is a circle with radius $\rho_{i}$ and position vector of centre denoted by $\mathbf{x}^{(i)}$, as shown in Figure 2 representing the intersection of a unit cell with the plane $x_{1}=0$. Centres $\mathbf{x}^{(i)}=\left(0, x_{2}^{(i)}, x_{3}^{(i)}\right)$ and radii $\rho_{i}$ of fibres $\Omega_{i}$ for $i=1, \ldots, 12$ are reported in Table 1 . It is assumed that the unit cell is symmetric with respect to the coordinate planes: thus, $\left|x_{k}^{(i+j)}\right|=\left|x_{k}^{(i)}\right|$ for $i=1,2,3, j=3,6,9$, and $k=1,2,3 . \mathbf{x}^{(i+j)}$ for $i=1,2,3$ is the position vector of the centres of the fibres in the volume characterized by $-\pi \leq x_{2} \leq 0$ and $0 \leq x_{3} \leq \pi$ for $j=3$ and the volume characterized by $-\pi \leq x_{2} \leq 0$ and $-\pi \leq x_{3} \leq 0$ for $j=6$. Matrix and fibres are linear elastic and isotropic; the interface between matrix and fibres is perfect. Young's modulus of the matrix is denoted by $E_{0}$ and is equal to one GPa; Young's modulus of the generic fibre $\Omega_{i}$ for $i=$ $1, \ldots, 12$ is denoted by $E_{i}$. Poisson's ratio of the matrix is $v_{0}$ and is equal to 0.35 . Poisson's ratio of the generic fibre $\Omega_{i}$ for $i=1, \ldots, 12$ is denoted by $v_{i}$. The geometrical and mechanical properties adopted for the fibres of the multiphase composite considered in this first example are reported in Table 1. It is noted that the considered composite is unidirectional and now the previous three-dimensional series (such as (13), (20), and (21)) applied to unidirectional composites reduce to twodimensional series by imposing $\zeta_{1}=0$.

In the following, the multiphase composite is subject to an average strain $\varepsilon_{0 i j}=\left(\delta_{i 1} \delta_{j 3}+\delta_{i 3} \delta_{j 1}\right) / 2$, where $\varepsilon_{0 i j}$ is the $i j$-component of the tensor $\varepsilon_{0}$ and $\delta_{i j}$ is the Kronecker delta. Figure 3 shows a three-dimensional surface, whose points are $\left(x_{2}, x_{3}, s_{110} \sigma_{13}\left(0, x_{2}, x_{3}\right)\right)$ for $\left|x_{2}\right| \leq \pi$ and $\left|x_{3}\right| \leq \pi$. The function $s_{110} \sigma_{13}\left(0, x_{2}, x_{3}\right)$ is the approximation (16) of the local stress $\sigma_{13}$ evaluated at the plane $x_{1}=0$ for $n=110$. In the 


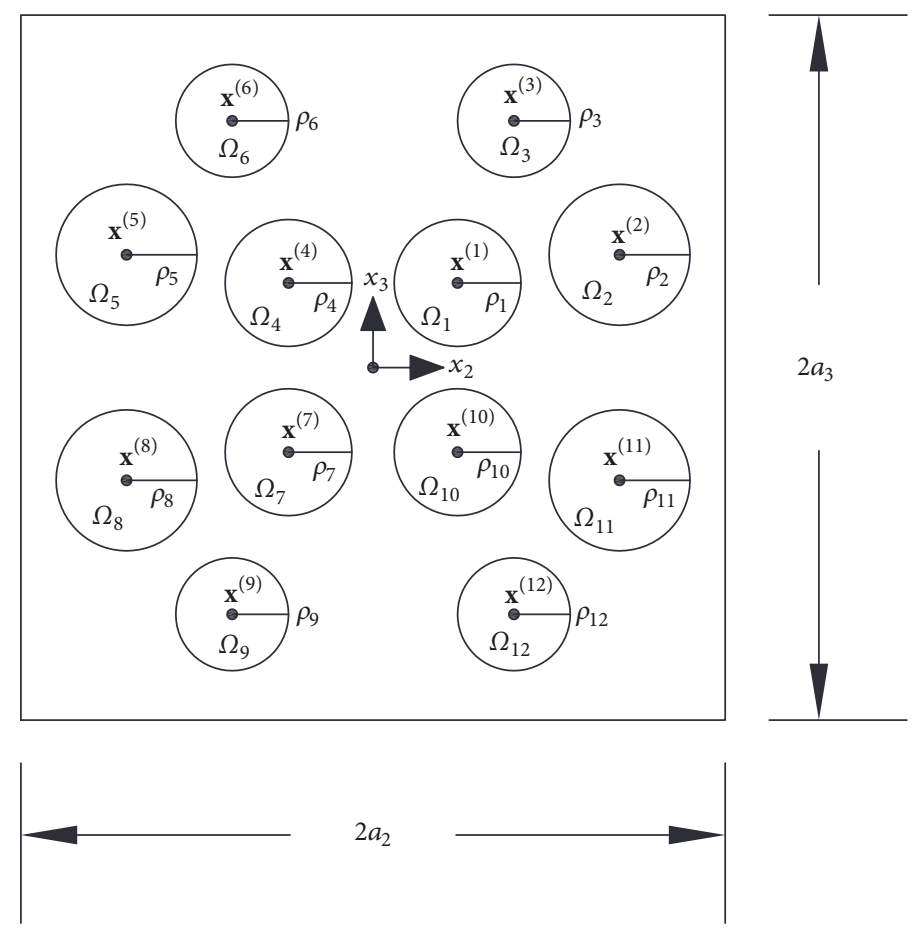

Figure 2: Fibres $\Omega_{i}$ for $i=1, \ldots, N_{f}$ in the unit cell of the multiphase composite with $N_{f}=12$.

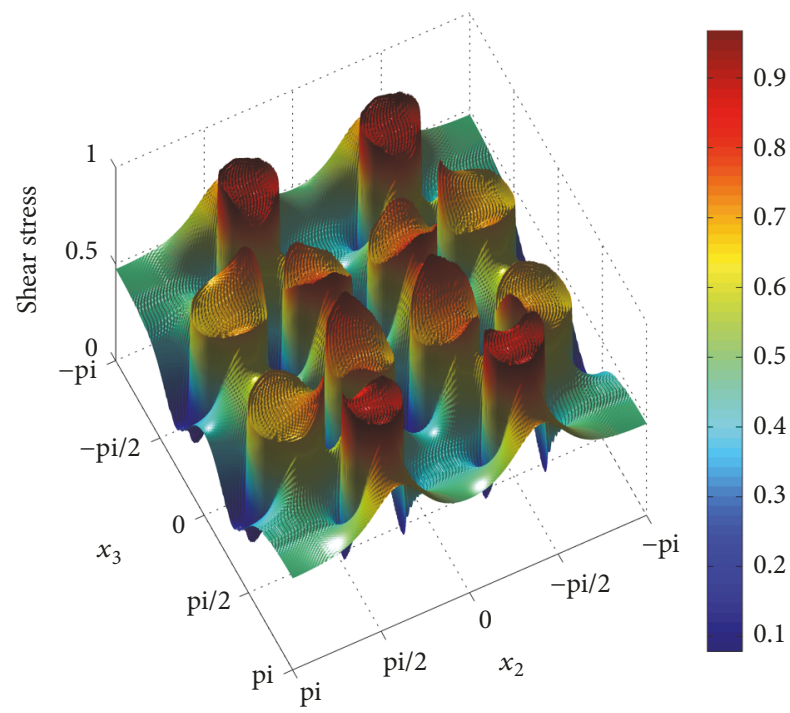

Figure 3: Partial sum stress $s_{110} \sigma_{13}(\mathbf{x})$ for the longitudinal shear stress of the multiphase composite.

figures of this work, the stress is in GPa and "pi" in the axes stands for $\pi$. Gibbs phenomenon is evident and large oscillations of the surface occur not only at the jump discontinuity along the fibre-matrix interface but also in the interior of the generic fibre and in some portion of the matrix. This behaviour is confirmed in Figure 4, where approximations (16) and (23) of the local stress $\sigma_{13}$ and $\sigma_{12}$ are plotted in different view sections for $n=110$. Specifically, Figures $4(\mathrm{a})$ and 4 (b) show the plots of stress approximations $s_{110} \sigma_{13}\left(0, x_{2}, x_{3}\right)$ and $s_{110} \sigma_{12}\left(0, x_{2}, x_{3}\right)$, respectively, for $\left|x_{2}\right| \leq \pi$ and $\left|x_{3}\right| \leq \pi$. Figures $4(\mathrm{c})$ and $4(\mathrm{~d})$ show the plots of $s_{110} \sigma_{13}\left(0, x_{2}, \pi / 4\right)$ and $s_{110} \sigma_{12}\left(0, x_{2}, \pi / 4\right)$, respectively, against $x_{2}$-axis for $\left|x_{2}\right| \leq$ $\pi$ : in other words, the approximations are plotted along the straight line segment with end points given by $(0,-\pi, \pi / 4)$ and $(0, \pi, \pi / 4)$. Figures $4(\mathrm{e})$ and $4(\mathrm{f})$ show the plots of stress approximations $s_{110} \sigma_{13}\left(0,3 \pi / 4, x_{3}\right)$ and $s_{110} \sigma_{12}\left(0,3 \pi / 4, x_{3}\right)$, respectively, against $x_{3}$-axis for $\left|x_{3}\right| \leq \pi$ : these approximations are plotted along the straight line segment with end points given by $(0,3 \pi / 4,-\pi)$ and $(0,3 \pi / 4, \pi)$. The iterated Fejér partial sum stress $\sigma_{110} \sigma_{13}(\mathbf{x})$ is also plotted in Figures $4(\mathrm{c})$ and $4(\mathrm{e}): \sigma_{110} \sigma_{13}(\mathbf{x})$ represents the approximation (23) of the local stress $\sigma_{13}$ for $n=110$. A similar description applies to $\sigma_{110} \sigma_{12}(\mathbf{x})$, which is plotted in Figures $4(\mathrm{~d})$ and $4(\mathrm{f})$. In the figure legends of this work, the iterated Fejér partial sum stress is denoted by "Iter. Fejér." In Figures 4(c)-4(f), the solutions provided by accurate FEM analyses are also plotted for comparison purpose and are denoted by the acronym "FEM" in the figure legends (FEM solution is plotted only for $0 \leq x_{2} \leq \pi$ in Figures 4(c) and 4(d)).

Figures 4(c)-4(f) show the iterated Fejér partial sum stress behaves better than the partial sum stress given by approximation (16). However, accuracy of the iterated Fejér partial sum stress is not satisfactory in some portions of the solids; see, e.g., the behaviour of the green curve in the matrix phase of Figure 4(c). In order to obtain better approximations of the local stress, means of summability methods have been introduced in Section 3 and are applied to the considered multiphase composite in Section 4.1. Relevant results are shown in Figure 5, where Riesz approximations (22) of the local stress $\sigma_{13}$ and $\sigma_{12}$ are plotted in different view sections for $n=110$. The values $\alpha=\gamma=1.8$ are adopted in 


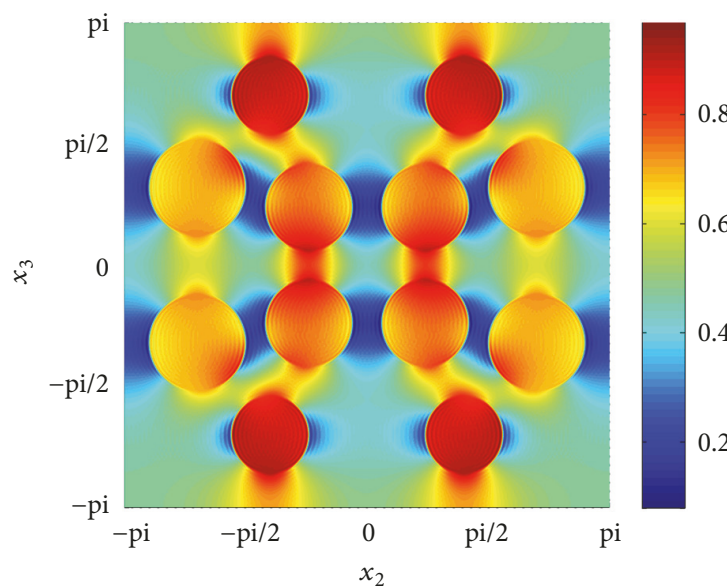

(a)

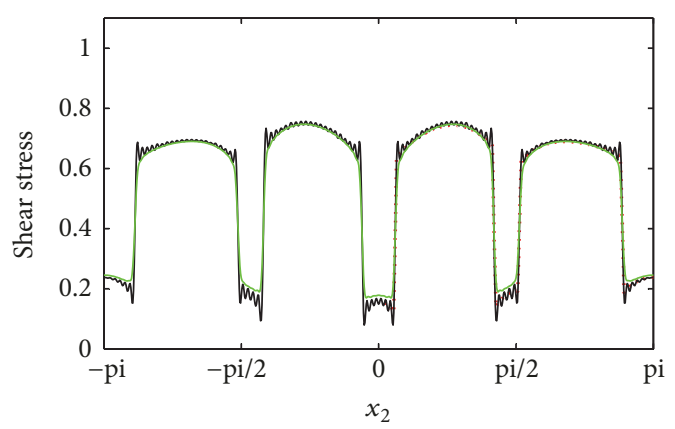

…. FEM

$-s_{110} \sigma_{13}$

- Iter. Fejér

(c)

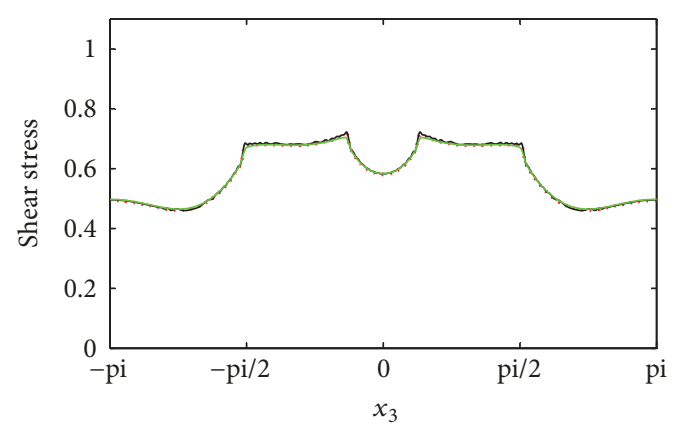

$\begin{array}{ll}\ldots . . . & \text { FEM } \\ - & s_{110} \sigma_{13} \\ & \text { Iter. Fejér }\end{array}$

(e)

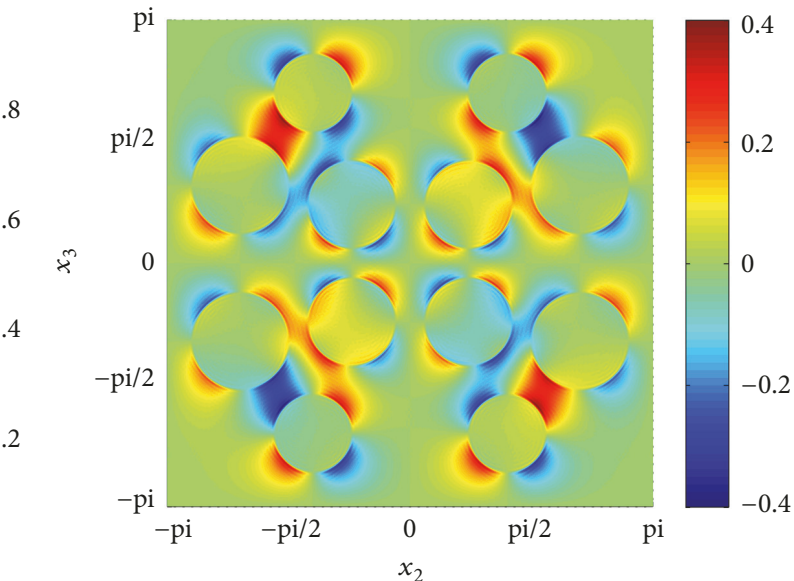

(b)
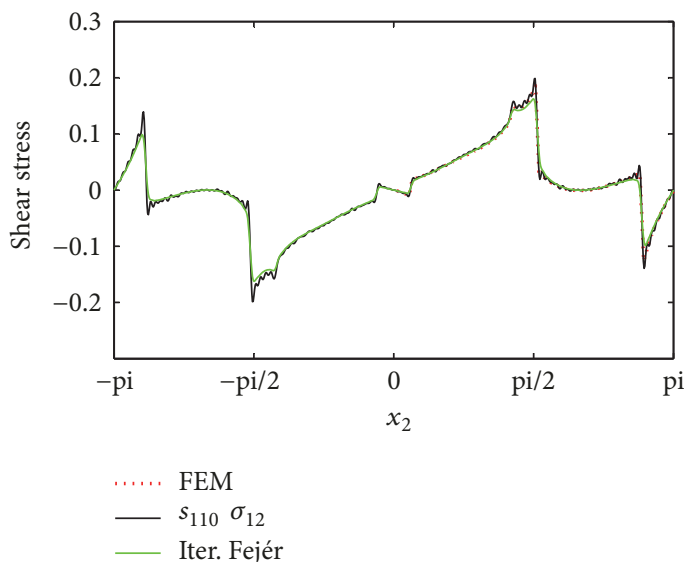

(d)

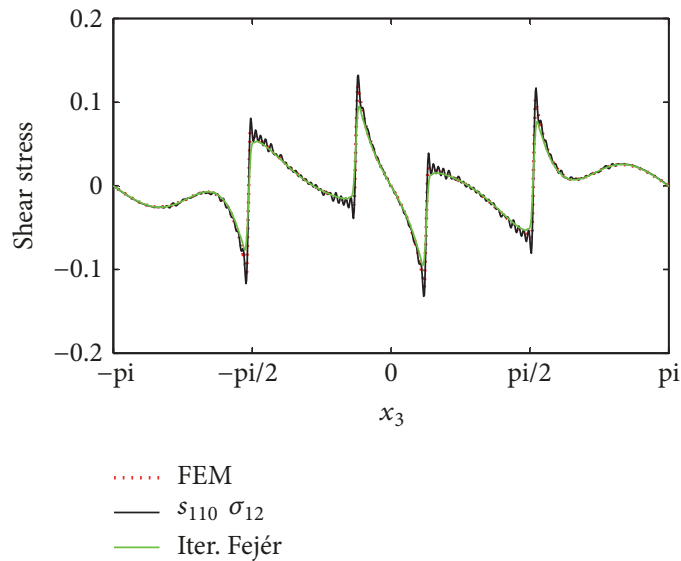

(f)

FIGURE 4: Gibbs phenomenon in partial sums and iterated Fejér partial sums for the local stress of the multiphase composite: (a) partial sum stress $s_{110} \sigma_{13}(\mathbf{x})$; (b) partial sum stress $s_{110} \sigma_{12}(\mathbf{x})$; (c) partial sum stress $s_{110} \sigma_{13}\left(0, x_{2}, \pi / 4\right)$ and iterated Fejér partial sum $\sigma_{110} \sigma_{13}\left(0, x_{2}, \pi / 4\right)$ along the $x_{2}$-axis; (d) partial sum stress $s_{110} \sigma_{12}\left(0, x_{2}, \pi / 4\right)$ and iterated Fejér partial sum $\sigma_{110} \sigma_{12}\left(0, x_{2}, \pi / 4\right)$ along the $x_{2}$-axis; (e) partial sum stress $s_{110} \sigma_{13}\left(0,3 \pi / 4, x_{3}\right)$ and iterated Fejér partial sum $\sigma_{110} \sigma_{13}\left(0,3 \pi / 4, x_{3}\right)$ along the $x_{3}$-axis; (f) partial sum stress $s_{110} \sigma_{12}\left(0,3 \pi / 4, x_{3}\right)$ and iterated Fejér partial sum $\sigma_{110} \sigma_{12}\left(0,3 \pi / 4, x_{3}\right)$ along the $x_{3}$-axis. 


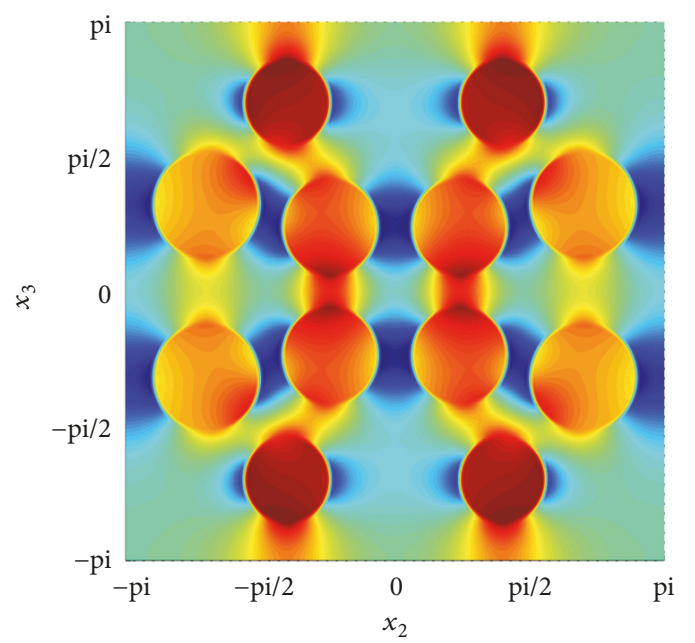

(a)
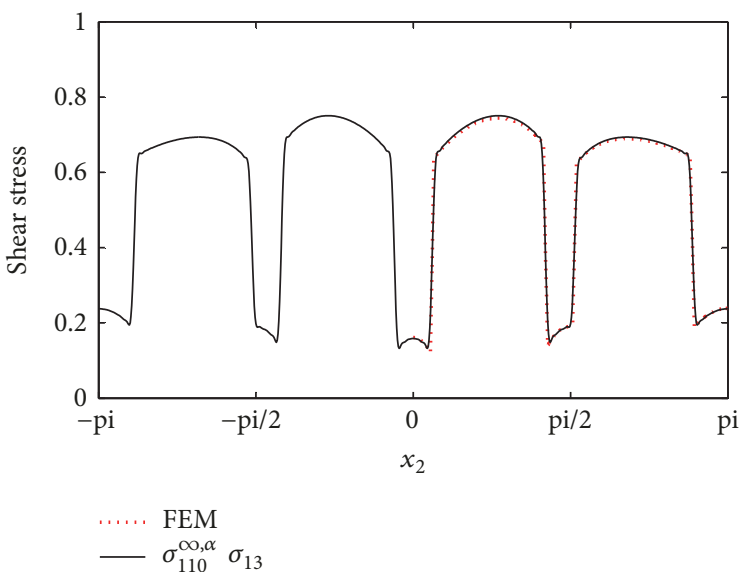

(c)

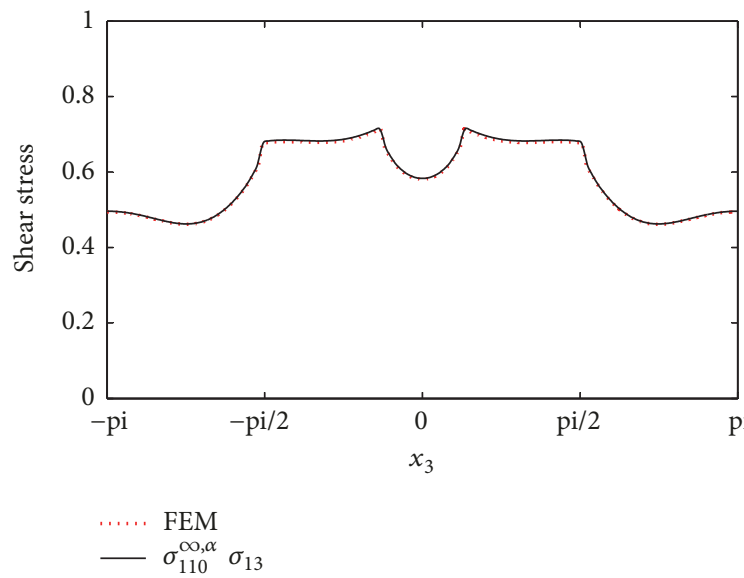

(e)

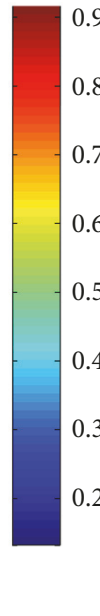

0.7
0.9

6.6

5

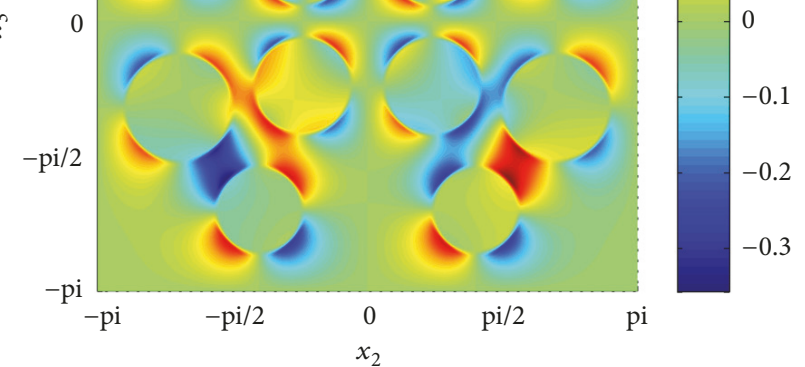

(b)

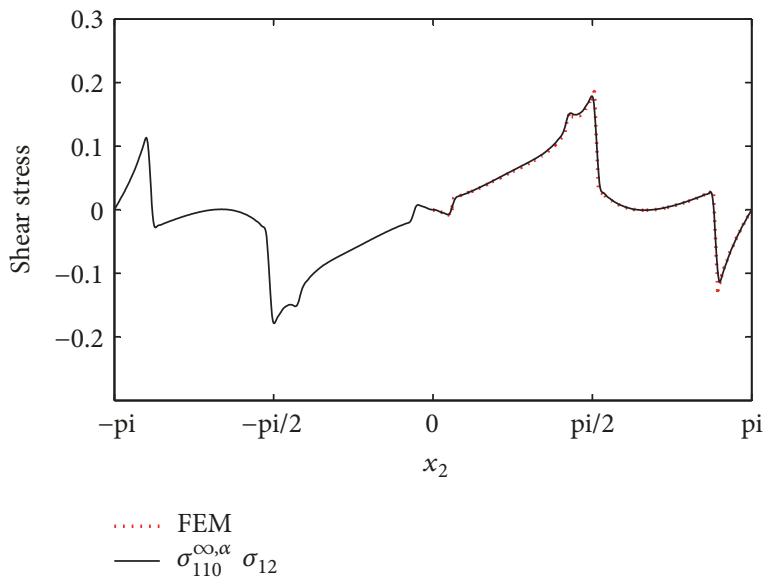

(d)

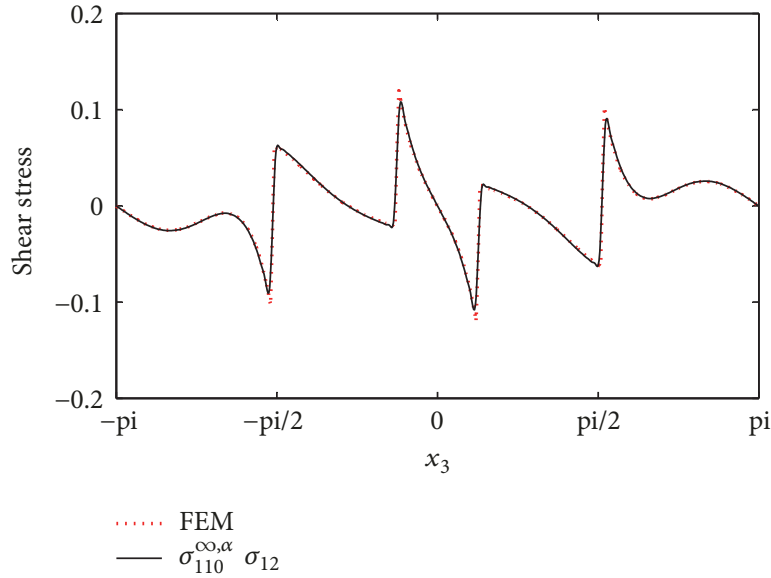

(f)

Figure 5: Riesz approximations of the local stress for the multiphase composite: (a) $\sigma_{110}^{\infty, \alpha} \sigma_{13}(\mathbf{x})$; (b) $\sigma_{110}^{\infty, \alpha} \sigma_{12}(\mathbf{x}) ;(\mathrm{c}) \sigma_{110}^{\infty, \alpha} \sigma_{13}\left(0, x_{2}, \pi / 4\right)$ along the $x_{2}$-axis; (d) $\sigma_{110}^{\infty}, \alpha \sigma_{12}\left(0, x_{2}, \pi / 4\right)$ along the $x_{2}$-axis; (e) $\sigma_{110}^{\infty, \alpha} \sigma_{13}\left(0,3 \pi / 4, x_{3}\right)$ along the $x_{3}$-axis; (f) $\sigma_{110}^{\infty, \alpha} \sigma_{12}\left(0,3 \pi / 4, x_{3}\right)$ along the $x_{3}$-axis.

the results of Section 4. Figures $5(\mathrm{a})$ and $5(\mathrm{~b})$ show the plots of $\sigma_{110}^{\infty, \alpha} \sigma_{13}\left(0, x_{2}, x_{3}\right)$ and $\sigma_{110}^{\infty, \alpha} \sigma_{12}\left(0, x_{2}, x_{3}\right)$, respectively, for $\left|x_{2}\right| \leq \pi$ and $\left|x_{3}\right| \leq \pi$. Figures 5(c) and 5(d) show the plots of stress approximations $\sigma_{110}^{\infty, \alpha} \sigma_{13}\left(0, x_{2}, \pi / 4\right)$ and $\sigma_{110}^{\infty, \alpha} \sigma_{12}\left(0, x_{2}, \pi / 4\right)$, respectively, against $x_{2}$-axis for $\left|x_{2}\right| \leq \pi$. Figures 5(e) and 5(f) show the plots of stress approximations $\sigma_{110}^{\infty, \alpha} \sigma_{13}\left(0,3 \pi / 4, x_{3}\right)$ and $\sigma_{110}^{\infty, \alpha} \sigma_{12}\left(0,3 \pi / 4, x_{3}\right)$, respectively, against $x_{3}$-axis for $\left|x_{3}\right| \leq \pi$. In Figures $5(\mathrm{c})-5(\mathrm{f})$, the 


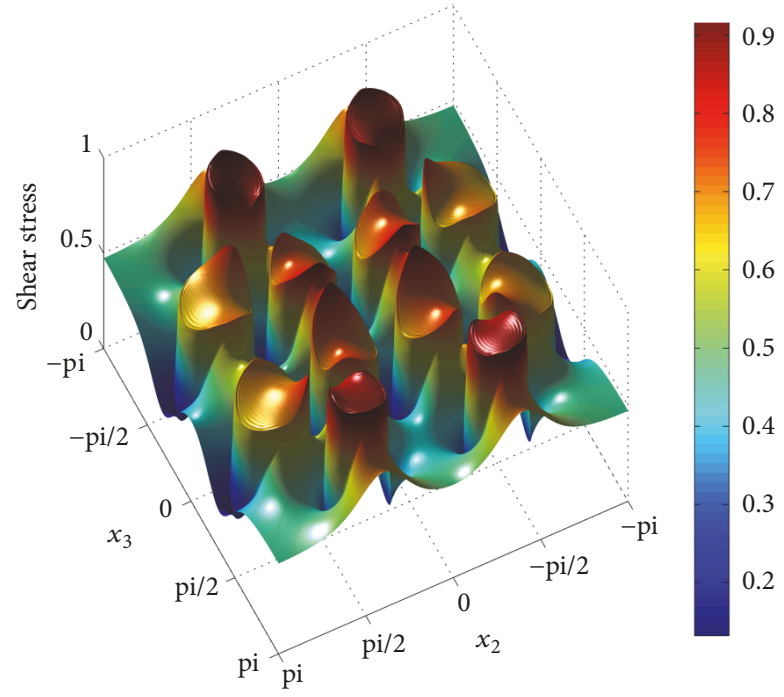

FIGURE 6: Riesz longitudinal shear stress $\sigma_{110}^{\infty, \alpha} \sigma_{13}(\mathbf{x})$ of the multiphase composite.

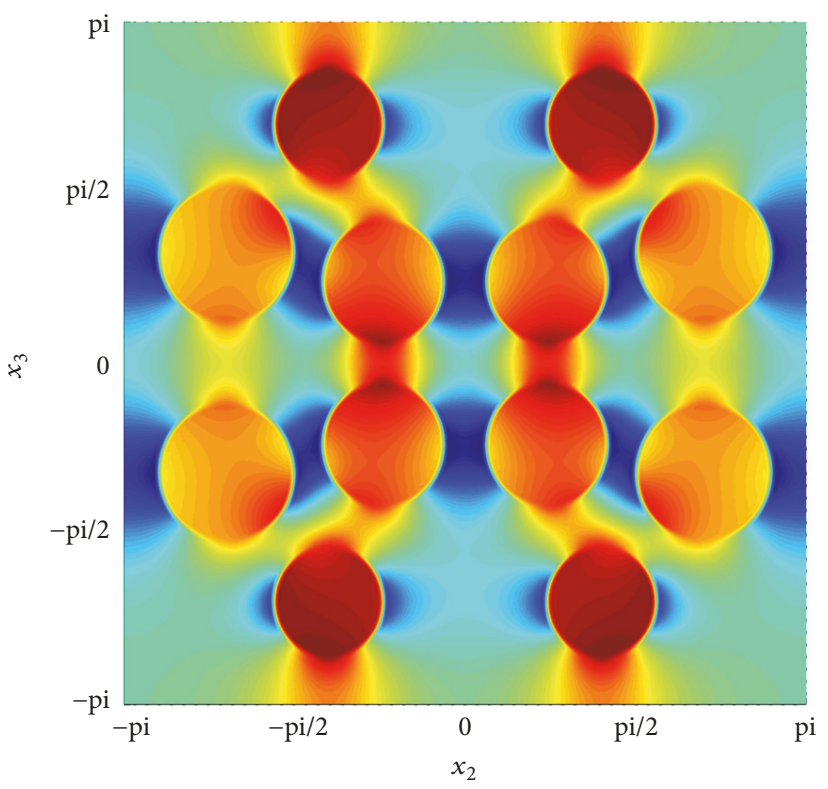

(a)
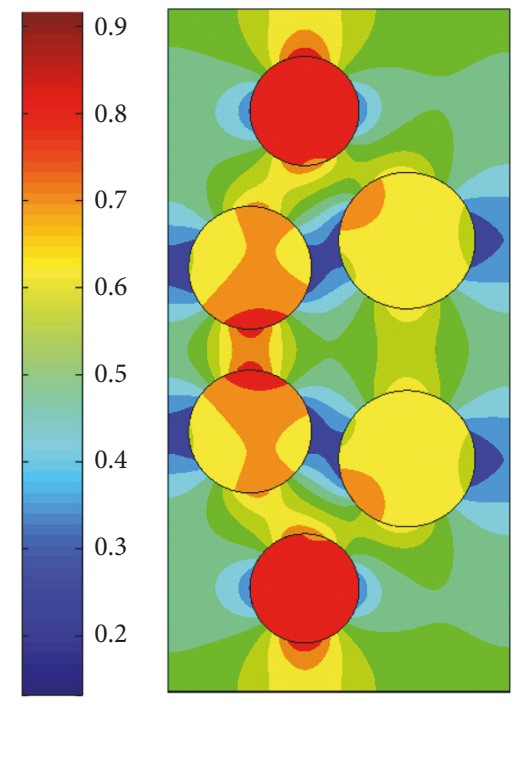

(b)
.11438
.202622
.290865
.379107
.46735
.555592
.643835
.732077
.82032
.908562

FIgURE 7: Comparison between Riesz mean and FEM solutions: (a) $\sigma_{110}^{\infty, \alpha} \sigma_{13}(\mathbf{x})$; (b) FEM approximation of $\sigma_{13}$.

solutions provided by accurate FEM analyses are also plotted for comparison purpose (FEM solution is plotted only for $0 \leq x_{2} \leq \pi$ in Figures 5(c) and 5(d)). Figure 5 shows Riesz means $\sigma_{110}^{\infty, \alpha} \boldsymbol{\sigma}(\mathbf{x})$ greatly reduce the Gibbs phenomenon and this also appears in Figure 6, which represents a threedimensional surface, whose points are $\left(x_{2}, x_{3}, \sigma_{110}^{\infty, \alpha} \sigma_{13}(0\right.$, $\left.\left.x_{2}, x_{3}\right)\right)$ for $\left|x_{2}\right| \leq \pi$ and $\left|x_{3}\right| \leq \pi$. Finally, Figure 7 illustrates a comparison between Riesz mean and FEM approximations: Figure 7(a) shows the plot of the Riesz mean $\sigma_{110}^{\infty, \alpha} \sigma_{13}\left(0, x_{2}, x_{3}\right)$ for $\left|x_{2}\right| \leq \pi$ and $\left|x_{3}\right| \leq \pi$; Figure $7(\mathrm{~b})$ shows the FEM approximation of the local stress $\sigma_{13}$ for $0 \leq x_{2} \leq \pi$ and $\left|x_{3}\right| \leq \pi$.
Figure 5 shows a good agreement between Riesz means and FEM solution. Some critical points appear at the fibrematrix interface mainly where the maximum shear stress occurs. In order to quantify a disagreement, the following percentage error is evaluated:

$$
e=\left|\frac{\tau_{F E M, \max }-\tau_{S, \max }}{\tau_{F E M, \max }}\right| \times 100
$$

where $\tau_{F E M, \text { max }}$ is the maximum shear stress provided by FEM solution in a given previous figure and $\tau_{S, \max }$ is the maximum value of the iterated Fejér partial sum stress or 


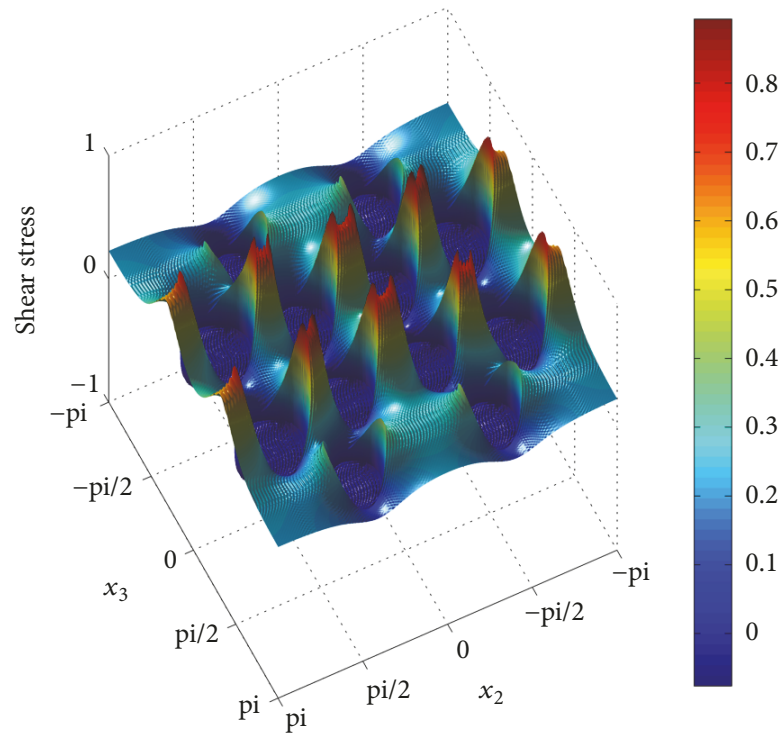

Figure 8: Partial sum stress $s_{110} \sigma_{13}(\mathbf{x})$ for the longitudinal shear stress of the material with voids.

TABLE 2: Percentage error $e$ in critical points of the multiphase composite.

\begin{tabular}{lcccc}
\hline & Figure 4(c) & Figure 5(c) & Figure 4(d) & Figure 5(d) \\
\hline$\tau_{S, \text { max }} \max \left(s_{110} \sigma_{13}\right)$ & $\max \left(\sigma_{110}^{\infty, \alpha} \sigma_{13}\right)$ & $\max \left(s_{110} \sigma_{12}\right)$ & $\max \left(\sigma_{110}^{\infty, \alpha} \sigma_{12}\right)$ \\
$e$ & 0.40 & 0.85 & 12.64 & 3.93 \\
\hline
\end{tabular}

the Riesz mean in the same figure where $\tau_{F E M \text {, max }}$ has been evaluated. For each of Figures 4(c), 4(d), 5(c), and 5(d), the percentage error $e$ is reported in Table 2, where the value adopted for $\tau_{S, \max }$ is also specified. For the shear stress $\sigma_{13}$, the percentage error is below $1 \%$ and the error provided by the iterated Fejér partial sum stress is less than the error provided by the Riesz mean, whose overall behaviour is better than iterated Fejér partial sum stress. For the shear stress $\sigma_{12}$, the Riesz mean provides a percentage error equal to $3.93 \%$. This relatively high value of $e$ occurs at some portions of the fibre-matrix interface, where the stress field has a jump discontinuity; elsewhere the Riesz mean performs better as shown in Figure 5. It is noted that the proposed Riesz means reduce not only the Gibbs phenomenon but also the percentage error: a greater value of $n$ in $\sigma_{n}^{q, \alpha} \boldsymbol{\sigma}(\mathbf{x})$ involves smaller values of the percentage error $e$. Therefore, a relatively high value of $e$ can be reduced by adopting larger values of $n$. On the other hand, the partial sum stress (16) does not exhibit this convergent behaviour at jump discontinuity.

Finally, an observation on the CPU memory usage is provided. In order to evaluate the partial sum stress $s_{n} \boldsymbol{\sigma}(\mathbf{x})$ or the Riesz mean $\sigma_{n}^{q, \alpha} \boldsymbol{\sigma}(\mathbf{x})$, one has to solve a system of $n_{S}$ scalar equations in $n_{S}$ scalar unknowns, where $n_{S}=2(n+1)^{2}$ thanks to polar symmetry and the symmetry of the unit cell with respect to the $x_{1}-x_{2}$ and $x_{1}-x_{3}$ coordinate planes. Therefore, the number of scalar equations is $n_{S}=24642$ for $n=110$. The number of equations in each of the accurate FEM analyses (which have been executed on half of the unit cell) is more than $1.5 \times 10^{5}$ : this value should not be compared with $n_{s}$ and is provided with the sole intent to highlight the refinement of the adopted FEM mesh and the accuracy of the relevant results.

4.2. Material with Voids. In this section, the approximations provided by partial sums, iterated Fejér partial sums, and Riesz means are compared with FEM solutions for a material containing voids. To this end, one should realize methods and models appropriate for a material with voids. In contrast with this rigorous approach, one can assume that inclusions with sufficiently small stiffness represent the voids. Following this approach, this section considers a heterogeneous material identical to that described in Section 4.1 except for $E_{i}=10^{-6}$ GPa for $i=1, \ldots, 12$. Fibres with such Young's moduli are practically voids embedded in the matrix. Figure 8 illustrates the Gibbs phenomenon in partial sum stress $s_{110} \sigma_{13}(\mathbf{x})$. Approximations provided by partial sums, iterated Fejér partial sums, and FEM simulations are compared with each other in Figure 9. Finally, the improvements of Riesz means are shown in Figures 10 and 11. For brevity, detailed descriptions of results presented in this section are omitted: symbols reported in captions and legends of Figures 8, 9, 10, and 11 have already been commented in Section 4.1.

\section{Conclusions}

In literature, methods based on Fourier series have been used to determine the global (averaged) properties of composite materials $[8,9,19,20]$. On the other hand, few works based on Fourier series have tried to evaluate the local fields, such as the strain and stress distribution in the material microstructure, and have found difficulties due to the Gibbs phenomenon. In this work, summability methods of threedimensional trigonometric Fourier series are applied in order to attenuate the Gibbs phenomenon occurring in local stress 


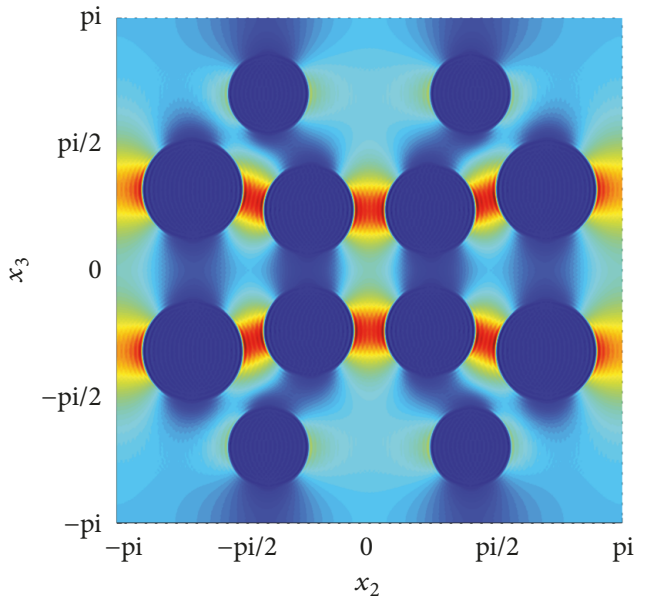

(a)

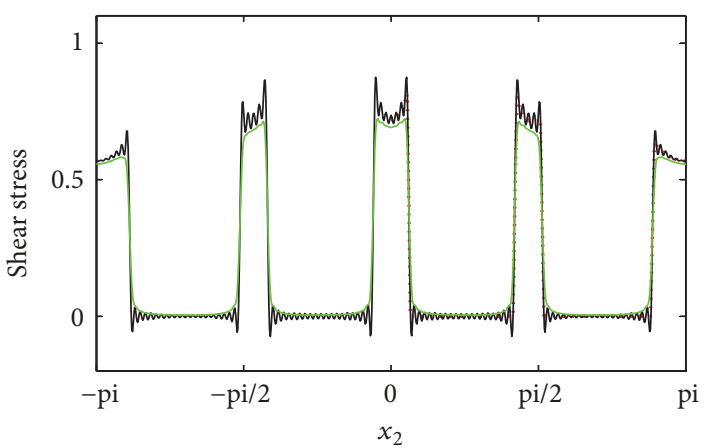

….. FEM

$-s_{110} \sigma_{13}$

— Iter. Fejér

(c)

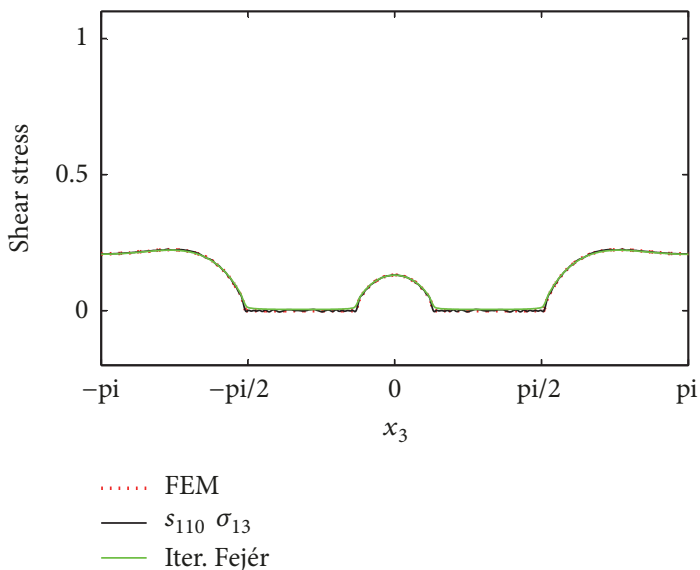

(e)

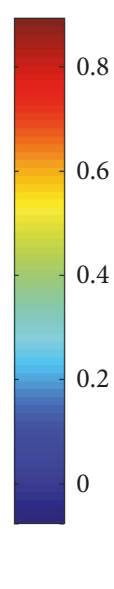

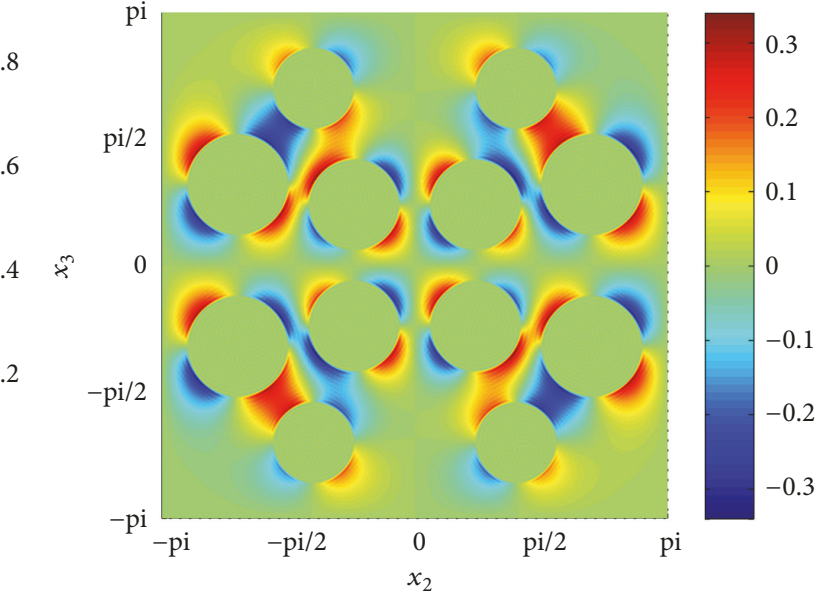

(b)

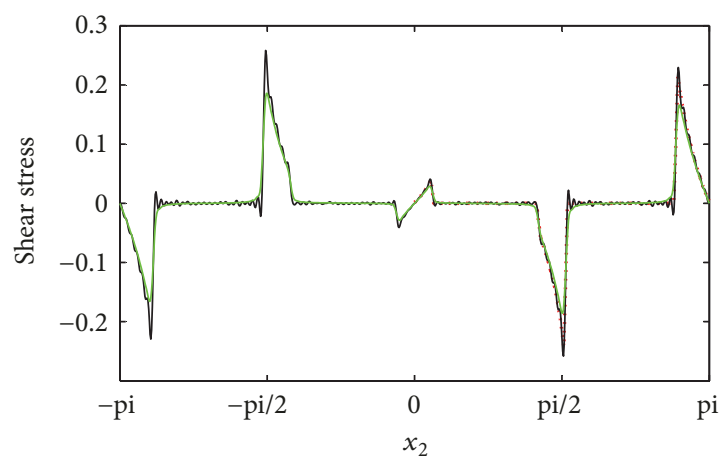

….. FEM

$-s_{110} \sigma_{12}$

— Iter. Fejér

(d)

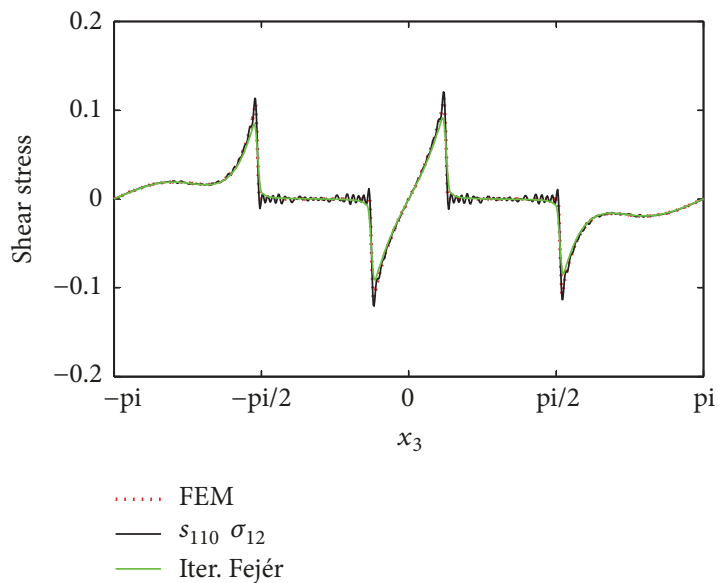

(f)

FIGURE 9: Gibbs phenomenon in partial sums and iterated Fejér partial sums for the local stress of the material with voids: (a) partial sum stress $s_{110} \sigma_{13}(\mathbf{x})$; (b) partial sum stress $s_{110} \sigma_{12}(\mathbf{x})$; (c) partial sum stress $s_{110} \sigma_{13}\left(0, x_{2}, \pi / 4\right)$ and iterated Fejér partial sum $\sigma_{110} \sigma_{13}\left(0, x_{2}, \pi / 4\right)$ along the $x_{2}$-axis; (d) partial sum stress $s_{110} \sigma_{12}\left(0, x_{2}, \pi / 4\right)$ and iterated Fejér partial sum $\sigma_{110} \sigma_{12}\left(0, x_{2}, \pi / 4\right)$ along the $x_{2}$-axis; (e) partial sum stress $s_{110} \sigma_{13}\left(0,3 \pi / 4, x_{3}\right)$ and iterated Fejér partial sum $\sigma_{110} \sigma_{13}\left(0,3 \pi / 4, x_{3}\right)$ along the $x_{3}$-axis; (f) partial sum stress $s_{110} \sigma_{12}\left(0,3 \pi / 4, x_{3}\right)$ and iterated Fejér partial sum $\sigma_{110} \sigma_{12}\left(0,3 \pi / 4, x_{3}\right)$ along the $x_{3}$-axis. 


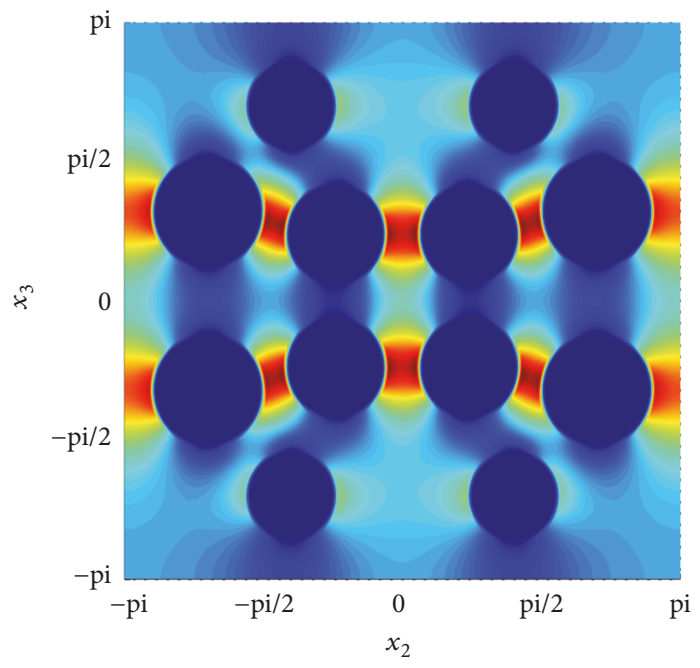

(a)
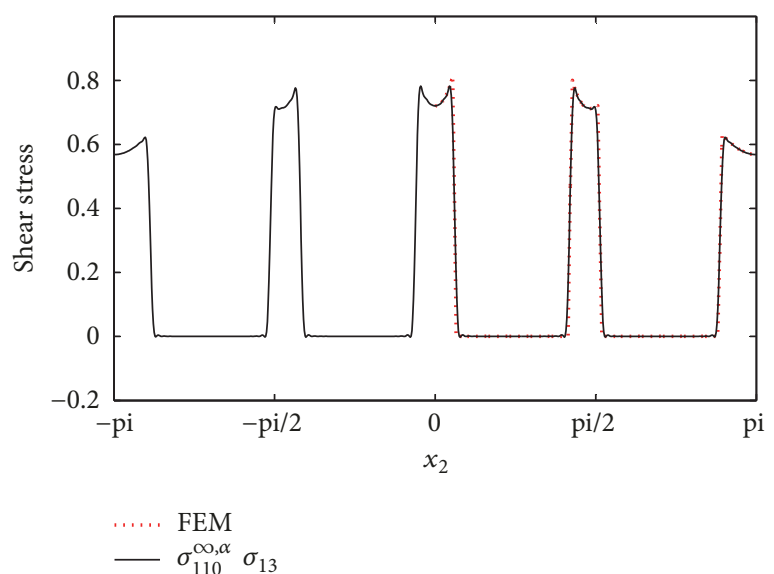

(c)

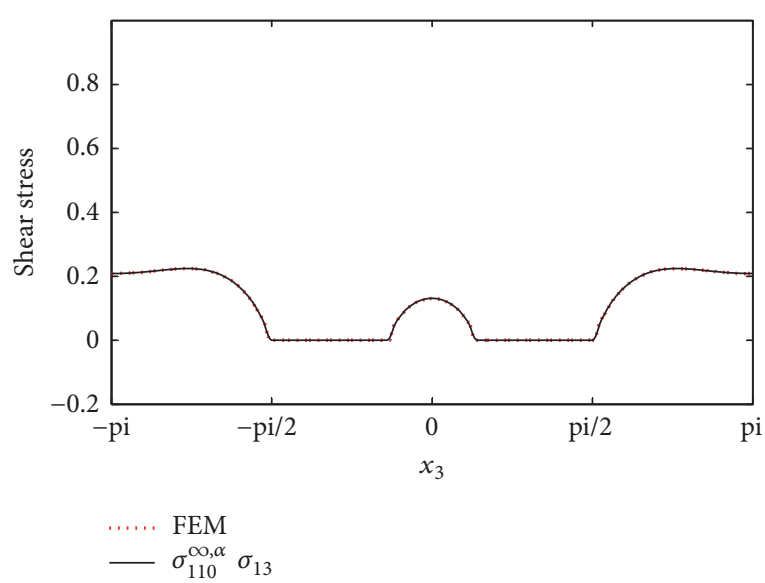

(e)

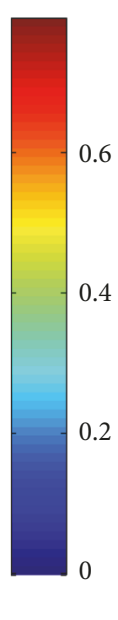

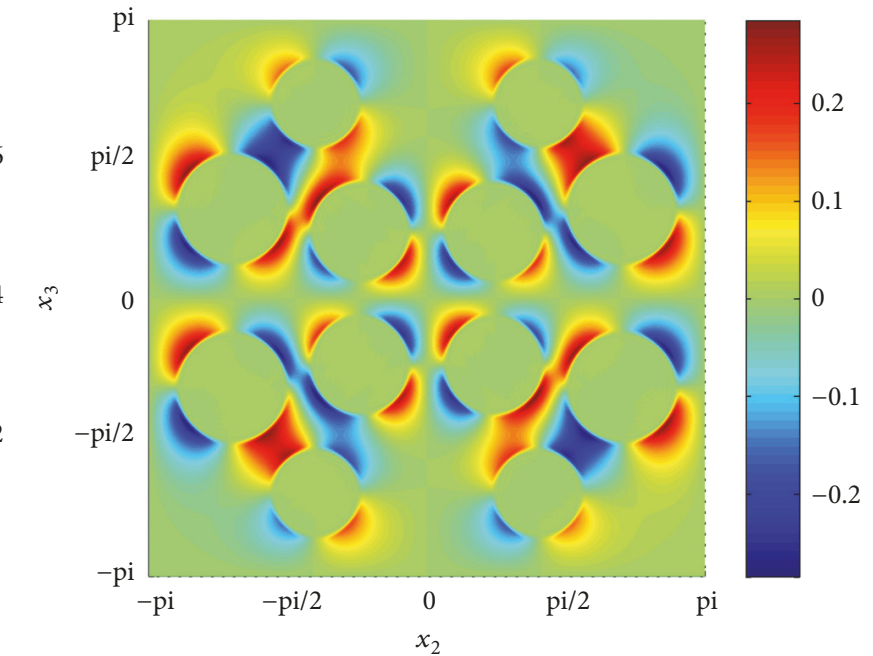

(b)

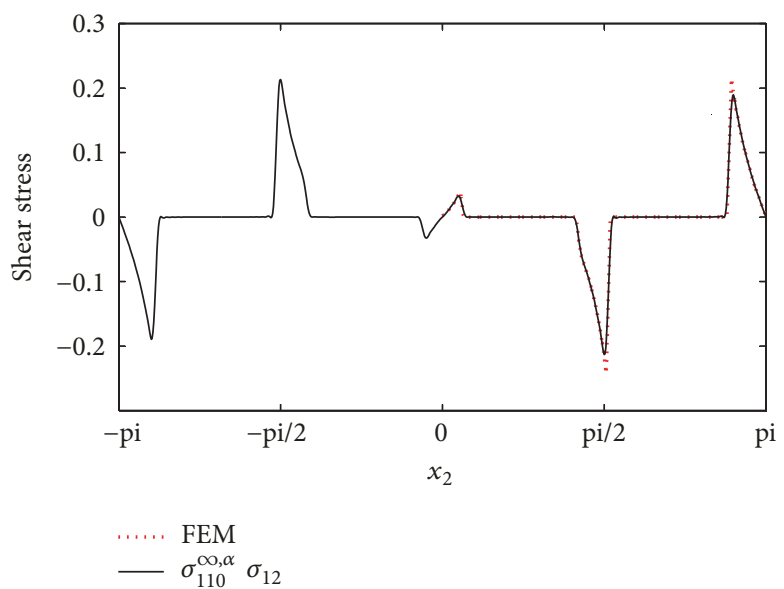

(d)

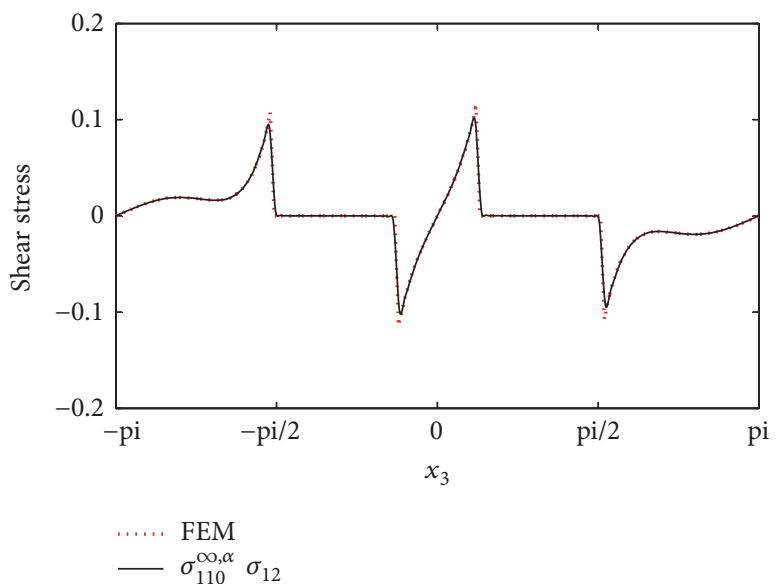

(f)

FIGURE 10: Riesz approximations of the local stress for the material with voids: (a) $\sigma_{110}^{\infty, \alpha} \sigma_{13}(\mathbf{x})$; (b) $\sigma_{110}^{\infty, \alpha} \sigma_{12}(\mathbf{x})$; (c) $\sigma_{110}^{\infty, \alpha} \sigma_{13}\left(0, x_{2}, \pi / 4\right)$ along the $x_{2}$-axis; (d) $\sigma_{110}^{\infty, \alpha} \sigma_{12}\left(0, x_{2}, \pi / 4\right)$ along the $x_{2}$-axis; (e) $\sigma_{110}^{\infty, \alpha} \sigma_{13}\left(0,3 \pi / 4, x_{3}\right)$ along the $x_{3}$-axis; (f) $\sigma_{110}^{\infty, \alpha} \sigma_{12}\left(0,3 \pi / 4, x_{3}\right)$ along the $x_{3}$-axis. 


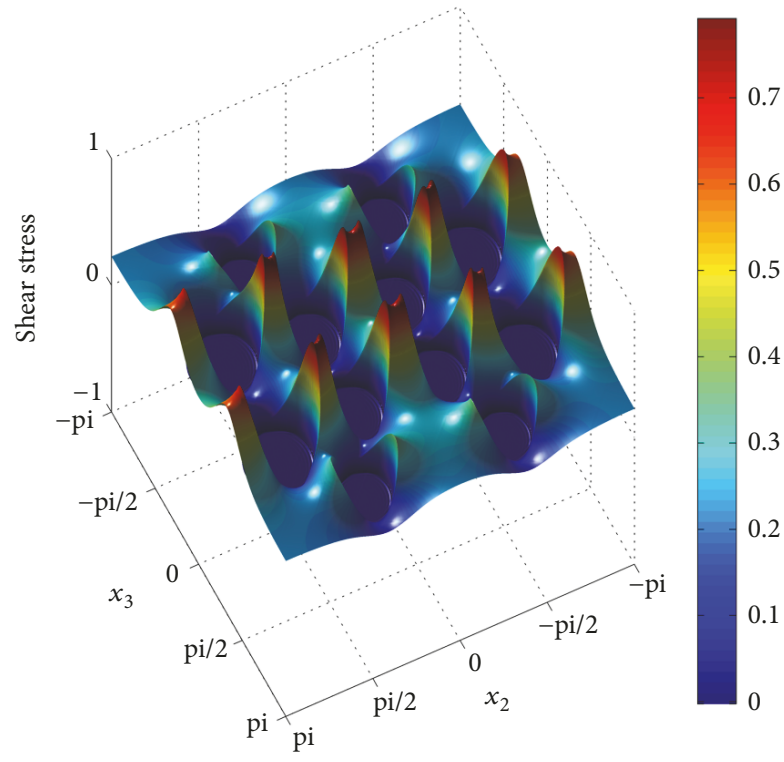

FIGURE 11: Riesz longitudinal shear stress $\sigma_{110}^{\infty, \alpha} \sigma_{13}(\mathbf{x})$ of the material with voids.

field in periodic heterogeneous materials characterized by unit cells containing multiple fibres or voids with different geometric and mechanical properties. An advantage of the proposed method is the easiness of modelling of the material microstructure through the functions (2), which admit closed-form expressions in many practical cases. Extensive numerical results are provided: partial sums, iterated Fejér partial sums, and Riesz means are compared with the solutions provided by accurate FEM analyses. The results show the effectiveness of the Riesz means, which have better convergence properties than those exhibited by partial sums and iterated Fejér partial sums.

\section{Data Availability}

The data used to support the findings of this study are available from the corresponding author upon request.

\section{Conflicts of Interest}

The author declares that there are no conflicts of interest regarding the publication of this paper.

\section{References}

[1] Hongwei Ying, Lisha Zhang, Kanghe Xie, and Dazhong Huang, "Pore Pressure Response to Groundwater Fluctuations in Saturated Double-Layered Soil," Mathematical Problems in Engineering, vol. 2015, Article ID 389089, 11 pages, 2015.

[2] Mousa Khalifa Ahmed, "Elastic buckling behaviour of a fourlobed cross section cylindrical shell with variable thickness under non-uniform axial loads," Mathematical Problems in Engineering, vol. 2009, Article ID 829703, 17 pages, 2009.

[3] Ali Golsoorat Pahlaviani and Suren Mkhitaryan, "Determination of the Stress State of a Piecewise Homogeneous Elastic Body with a Row of Cracks on an Interface Surface Subject to Antiplane Strains with Inclusions at the Tips," Mathematical
Problems in Engineering, vol. 2015, Article ID 851548, 22 pages, 2015.

[4] G. Wang and M.-J. Pindera, "Locally-exact homogenization of unidirectional composites with coated or hollow reinforcement," Materials \& Design, vol. 93, pp. 514-528, 2016.

[5] Lijie Li, Haichao Li, Fuzhen Pang, Xueren Wang, Yuan Du, and Shuo Li, "The Modified Fourier-Ritz Approach for the Free Vibration of Functionally Graded Cylindrical, Conical, Spherical Panels and Shells of Revolution with General Boundary Condition," Mathematical Problems in Engineering, vol. 2017, Article ID 9183924, 32 pages, 2017.

[6] F. Greco, "A study of stability and bifurcation in micro-cracked periodic elastic composites including self-contact," International Journal of Solids and Structures, vol. 50, no. 10, pp. 16461663, 2013.

[7] L. Leonetti, F. Greco, P. Trovalusci, R. Luciano, and R. Masiani, "A multiscale damage analysis of periodic composites using a couple-stress/Cauchy multidomain model: Application to masonry structures," Composites Part B: Engineering, vol. 141, pp. 50-59, 2018.

[8] S. Nemat-Nasser and M. Hori, Micromechanics: Overall Properties of Heterogeneous Materials, vol. 37 of North-Holland Series in Applied Mathematics and Mechanics, North-Holland Publishing Co., Amsterdam, 1st edition, 1993.

[9] R. Luciano and E. J. Barbero, "Formulas for the stiffness of composites with periodic microstructure," International Journal of Solids and Structures, vol. 31, no. 21, pp. 2933-2944, 1994.

[10] S. Kaßbohm, W. H. Müller, and R. Feßler, "Improved approximations of Fourier coefficients for computing periodic structures with arbitrary stiffness distribution," Computational Materials Science, vol. 37, no. 1-2, pp. 90-93, 2006.

[11] A. Caporale, L. Feo, and R. Luciano, "Eigenstrain and Fourier series for evaluation of elastic local fields and effective properties of periodic composites," Composites Part B: Engineering, vol. 81, pp. 251-258, 2015.

[12] A. Caporale, R. Luciano, C. M. Medaglia, and R. Penna, "Local stress in periodic composites via the Riesz summability method," Composites Part B: Engineering, vol. 150, pp. 27-35, 2018.

[13] G. Gát, "Pointwise convergence of cone-like restricted two-dimensional $(\mathrm{C}, 1)$ means of trigonometric Fourier series," Journal of Approximation Theory, vol. 149, no. 1, pp. 74-102, 2007.

[14] G. Gát, "Convergence of sequences of two-dimensional Fejér means of trigonometric FOUrier series of integrable functions," Journal of Mathematical Analysis and Applications, vol. 390, no. 2, pp. 573-581, 2012.

[15] F. Weisz, " $\ell 1$-summability of higher-dimensional Fourier series," Journal of Approximation Theory, vol. 163, no. 2, pp. 99116, 2011.

[16] F. Weisz, "Marcinkiewicz-summability of multi-dimensional Fourier transforms and Fourier series," Journal of Mathematical Analysis and Applications, vol. 379, no. 2, pp. 910-929, 2011.

[17] F. Weisz, "Summability of multi-dimensional trigonometric Fourier series," Surveys in Approximation Theory, vol. 7, pp. 1$179,2012$.

[18] V. L. Shapiro, Fourier Series in Several Variables with Applications to Partial Differential Equations, 2011. 
[19] A. Caporale, R. Luciano, and R. Penna, "Fourier series expansion in non-orthogonal coordinate system for the homogenization of linear viscoelastic periodic composites," Composites Part B: Engineering, vol. 54, no. 1, pp. 241-245, 2013.

[20] Q.-D. To, S.-T. Nguyen, G. Bonnet, and M.-N. Vu, "Overall viscoelastic properties of $2 \mathrm{D}$ and two-phase periodic composites constituted of elliptical and rectangular heterogeneities," European Journal of Mechanics - A/Solids, vol. 64, pp. 186-201, 2017. 


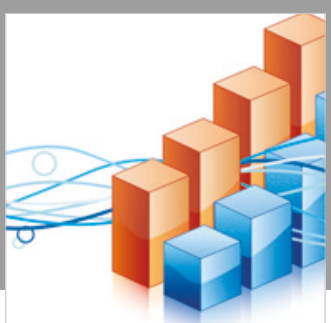

Advances in

Operations Research

\section{-n-m}
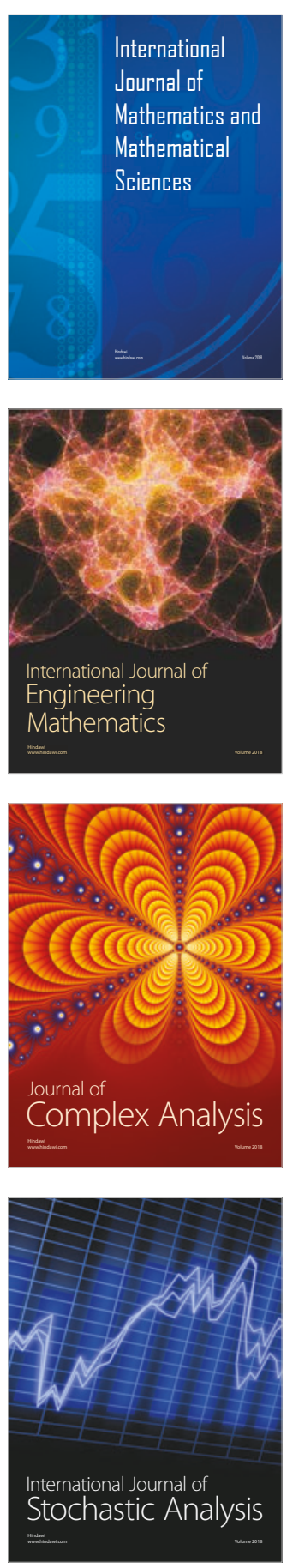
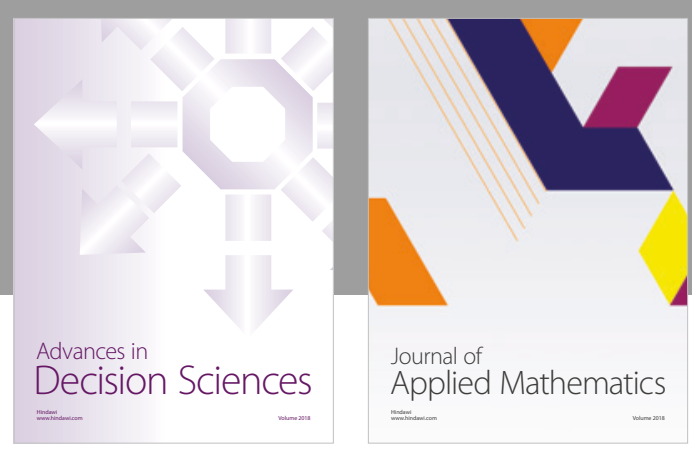

Journal of

Applied Mathematics
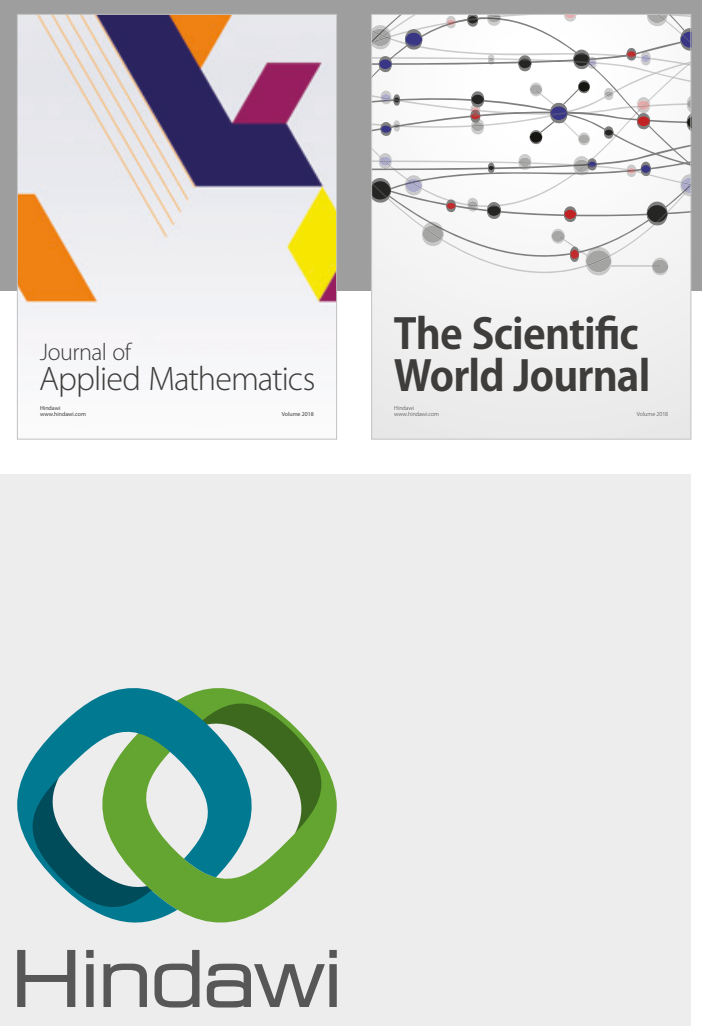

Submit your manuscripts at

www.hindawi.com

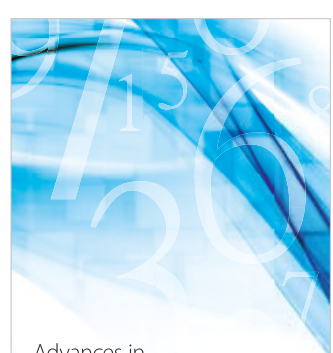

Advances in
Numerical Analysis
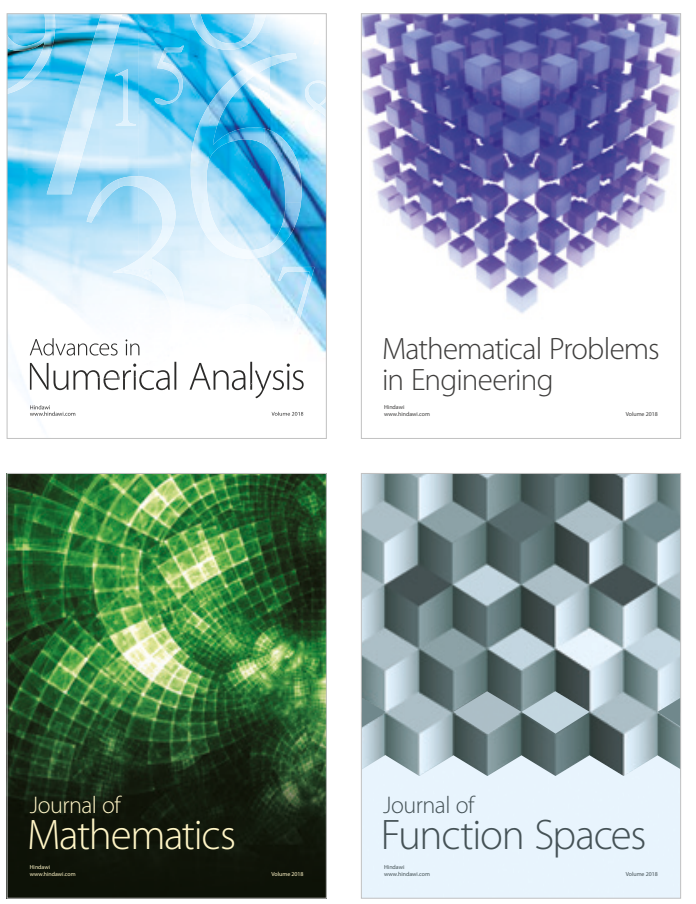

Mathematical Problems in Engineering

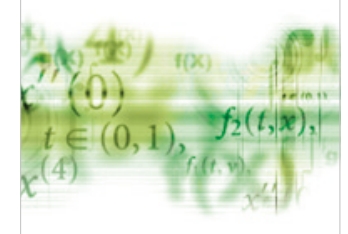

International Journal of

Differential Equations

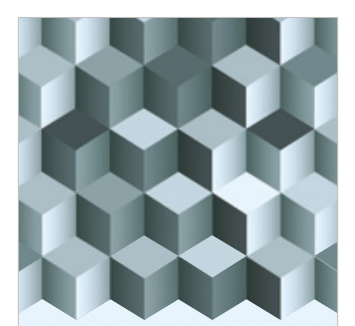

Journal of

Function Spaces

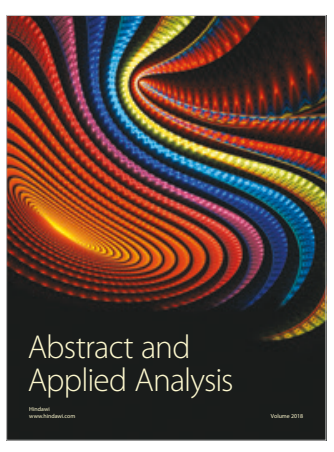

The Scientific

World Journal

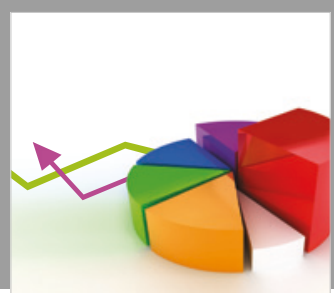

Journal of

Probability and Statistics
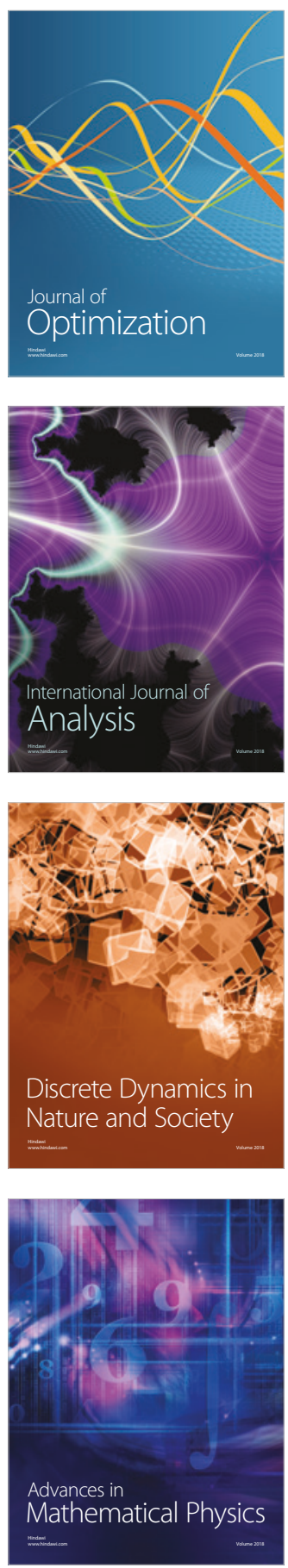